\title{
Guide de pratique clinique fondé sur des données probantes pour l'examen périodique de la vue chez les enfants de 0 à 5 ans au Canada
}

\author{
Comité mixte d'experts sur les guides de pratique clinique de l'Association canadienne \\ des optométristes et de la Société canadienne d'ophtalmologie : \\ ${ }^{1}$ Représentant la Société canadienne d'ophtalmologie. \\ ${ }^{2}$ Représentant l'Association canadienne des optométristes \\ ${ }^{3}$ Représentant l'Association canadienne d'ophtalmologie et de strabisme pédiatriques
}

\author{
Walter T. Delpero, \\ MD FRCSC - coprésident ${ }^{1}$ \\ Professeur adjoint \\ Université d'Ottawa \\ Département d'ophtalmologie

Barbara E. Robinson,
OD MPH PhD FAAO -
coprésidente ${ }^{2}$
Professeure émérite
École d'optométrie et de
sciences de la vision
Université de Waterloo

Jane A. Gardiner, MD FRCSC ${ }^{1,3}$ Professeure clinique Département d'ophtalmologie et des sciences de la vision Université de la Colombie-Britannique British Columbia Children's Hospital

Louise Nasmith, MDCM FCFP FRCPSC(Hon)

Professeure

Département de médecine familiale Université de la Colombie-Britannique

\section{Anne Rowan-Legg, MD FRCPC \\ Pédiatre-conseil Centre hospitalier pour enfants de l'est de l'Ontario et Hôpital Victoria de Renfrew Professeure adjointe Université d'Ottawa Département de pédiatrie}

Benoît Tousignant, OD MSc MPH FAAO ${ }^{2}$ Professeur adjoint École d'optométrie Université de Montréal

\section{RÉSUMÉ}

Contexte : Étant donné que les maladies oculaires avant l'âge de 5 ans sont courantes, une certaine forme de dépistage des troubles de la vision devrait être effectuée chez les enfants avant qu'ils ne fréquentent l'école primaire. Cependant, l'absence de recommandations nationales cohérentes crée de la confusion chez les patients, les professionnels des soins oculovisuels et les gouvernements.

Méthodes : L'objectif de ce document est de fournir des recommandations quant aux types d'examens oculaires à pratiquer chez les enfants en bonne santé de 0 à 5 ans ainsi que sur le moment et la périodicité de tels examens. Une recension des écrits a produit 403 articles. Un comité d'experts multidisciplinaire (composé de deux optométristes, d'un ophtalmologiste effectuant des examens complets de la vue, d'un ophtalmologiste pratiquant en pédiatrie, d'un médecin de famille et d'un pédiatre) a établi de façon indépendante les articles jugés essentiels à la question clinique. Les articles se prêtant à un classement [n=16] ont ensuite été soumis à une évaluation critique indépendante par un groupe externe, lequel a fourni un profil «GRADE » des articles à utiliser et leur a attribué une cote.

Recommandations : En plus du dépistage de routine effectué par les professionnels de première ligne, un examen complet de la vue mené par un professionnel possédant l'expertise nécessaire à la détection des facteurs de risque de l'amblyopie (comme un ophtalmologiste ou un optométriste) est requis durant la petite enfance. Les conclusions confirment l'importance de la détection précoce de l'amblyopie avant 36 mois et au plus tard 48 mois par le dépistage assorti d'au moins un examen complet de la vue avant l'âge de 5 ans.

Conclusions : Le dépistage de la vue effectué chez les bébés et les enfants par les fournisseurs de soins de première ligne au cours des consultations de routine et des vaccinations périodiques est un élément essentiel de la détection des maladies oculaires. Toutefois, le potentiel de détection précoce est limité et un examen oculovisuel complet est également recommandé avant que l'enfant n'entre à l'école. Si l'amblyopie, le strabisme ou une autre pathologie oculaire est détecté ou soupçonné, et que le problème dépasse le champ de compétences du professionnel qui examine le patient, celui-ci peut être dirigé vers le spécialiste approprié, ce qui permet d'amorcer le traitement en temps opportun.

\section{MOTS CLÉS :}

amblyopie, enfants, guide de pratique clinique, examen complet de la vue, nourrissons, examen périodique de la vue, soins de première ligne, erreur de réfraction, strabisme, dépistage des troubles de la vue 


\section{INTRODUCTION}

Le dépistage des troubles de la vision et les examens complets de la vue sont recommandés tout au long du cycle de vie en tant que moyen de déceler des maladies oculaires traitables qui, autrement, passeraient inaperçues ${ }^{1-5}$. Comme les troubles oculaires avant l'âge de 5 ans sont fréquents, les médecins de famille, les pédiatres, les optométristes et les ophtalmologistes insistent depuis longtemps pour effectuer un certain type de dépistage chez les enfants avant leur entrée à l'école (tableau 1) 1,3-9. Les recommandations sur le dépistage formulées par les organismes nationaux sont plurielles sans compter qu'elles varient d'une province à l'autre ainsi qu'à l'intérieur des provinces, des pays, voire des commissions scolaires. La Société canadienne d'ophtalmologie (SCO) et l'Association canadienne des optométristes (ACO) ont reconnu que l'absence de recommandations nationales cohérentes de la part des ophtalmologistes et des optométristes au sujet de la périodicité du dépistage et des examens complets de la vue créait de la confusion chez les patients, les professionnels des soins oculovisuels et les gouvernements. Elles ont aussi reconnu que les guides de pratique sur les soins oculovisuels devraient inclure les commentaires des autres principaux professionnels qui participent à la surveillance médicale des enfants en première ligne, à savoir les pédiatres et les médecins de famille. La SCO et l'ACO ont ainsi invité le Collège des médecins de famille du Canada (CMFC) et la Société canadienne de pédiatrie (SCP) à nommer chacun un représentant à un comité interdisciplinaire d'experts en guides de pratique chargé de formuler des recommandations fondées sur des données probantes et sur l'expertise clinique et les réalités de la pratique de tous les représentants.

Idéalement, les guides de pratique sont des outils laissant place à la flexibilité qui sont basés sur les meilleures preuves scientifiques disponibles. Ils reflètent aussi un consensus de professionnels du milieu concerné et permettent aux professionnels de la santé de se servir de leur jugement dans la prise en charge des patients ${ }^{10}$. Les guides de pratique ne fournissent pas de « recette » dans la pratique de la médecine ni des soins de santé et ils ne remplacent pas le jugement clinique $^{11}$; ils complètent plutôt les modèles de pratique. Ce guide de pratique devrait être considéré dans ce contexte. Il ne donnera pas nécessairement de bons résultats dans tous les cas. Par ailleurs, il ne vise pas à définir ni à servir une norme légale de soins de santé12 et ne doit pas être utilisé en tant que source légale du fait que sa nature générale ne permet pas d'offrir des recommandations individualisées pour tous les patients et pour toute circonstance ${ }^{11}$. Les normes de soins médicaux s'appliquent précisément à tous les faits et circonstances propres à un cas particulier et peuvent être sujettes à des changements à mesure que les connaissances scientifiques, la technologie et les modèles de pratique progressent. En effet, les professionnels de la santé doivent tenir compte des besoins, des préférences, des valeurs et de la situation financière et personnelle de chaque patient de même que travailler dans le contexte de leur milieu de soins.

L'objectif de ce document est de fournir des recommandations quant aux types d'examens oculaires à pratiquer chez les enfants en bonne santé de 0 à 5 ans (p. ex. non prématurés, sans maladie systémique chronique comme le diabète, sans perte auditive ni troubles neurodéveloppementaux) ainsi que sur le moment et la périodicité de tels examens. Le public cible se compose de tous les professionnels de la santé canadiens qui dirigent ou voient des nourrissons et des enfants pour un examen de la vue (c.-à-d. pédiatres, médecins de famille, fournisseurs de soins de première ligne, ophtalmologistes et optométristes, infirmières et infirmières praticiennes). La périodicité recommandée des examens intéressera également le grand public et les décideurs. Il est reconnu qu'il y a des inégalités dans les ressources humaines, financières et de santé dans les différentes régions du pays et que ces facteurs ont une incidence sur les options et les décisions des professionnels de la santé et des patients. Dans une certaine mesure, ce guide de pratique pourrait servir à promouvoir les soins oculovisuels de base pour la population pédiatrique dans les régions mal desservies.

\section{MÉTHODES}

Ce guide de pratique a été élaboré systématiquement à partir de l'examen approfondi des écrits médicaux et de l'expérience clinique des professionnels de la santé interdisciplinaires siégeant au Comité d'experts. Dans la mesure du possible, le contenu de ce document a été élaboré conformément au Guide sur les guides de pratique clinique ${ }^{11}$ et les paramètres correspondant aux six domaines de l'instrument Appraisal of Guidelines Research and Evaluation II $(A G R E E I I)^{13,14}$. Ces domaines couvrent les dimensions suivantes des guides de pratique : portée et objectif, participation des personnes concernées, rigueur du développement, clarté et présentation, applicabilité et indépendance des rédacteurs. De plus, la liste de vérification pour l'élaboration du guide de pratique par Schünemann et ses collaborateurs a été consultée et mise en application le cas échéant ${ }^{15}$.

La question clinique clé de ce guide est la suivante : «Quels sont les meilleurs moments et la meilleure périodicité des examens oculaires chez les enfants âgés de 0 à 5 ans afin de promouvoir une santé oculovisuelle optimale? » Pour répondre à cette question, des recherches ont été effectuées par une bibliothécaire médicale dans PubMed/ Medline (1995 à avril 2018) à l'aide d'un vocabulaire et de mots clés contrôlés et appropriés (« amblyopie, erreur 
de réfraction, dépistage des troubles de la vision, strabisme » combinés à des variantes du terme « examen complet de la vue »). Ces recherches ont été corroborées par des recherches par échantillonnage menées dans EMBASE, Web of Science et la Bibliothèque Cochrane. Les recherches se sont limitées aux enfants et aux nourrissons de 0 à 5 ans et aux écrits publiés dans des revues à comité de lecture rédigés en anglais ou en français. Toutes les études ont été incluses dans la recherche d'essais cliniques et d'études d'observation correctement menées; des études sur l'amblyopie, les facteurs de risque de l'amblyopie et les erreurs de réfraction; des études portant sur les soins de première ligne et les infrastructures fondées sur la population; des études sur les tests de dépistage offerts dans les établissements de soins de première ligne (p. ex., tests d'acuité visuelle, du reflet rétinien et de l'écran) ou des techniques d'examen utilisées par les optométristes et les ophtalmologistes (rétinoscopie, etc.); et des études portant sur les résultats suivants : amélioration de l'acuité visuelle, diminution de l'amblyopie, meilleur rendement scolaire et meilleure qualité de vie. Les résultats ne se sont pas limités aux recensions systématiques, aux essais aléatoires ou cliniques avec groupe témoin et aux études d'observation. Les recherches ont été périodiquement actualisées et les bibliographies des études incluses vérifiées pour trouver d'autres références aux études et articles pertinents. (Les stratégies de recherche sont disponibles à l'annexe 1.) (Les critères d'inclusion et d'exclusion figurent à l'annexe 2.)

La recension des écrits a produit 403 articles. Les membres du Comité ont été invités à passer en revue le résumé des articles et à indiquer de façon indépendante les articles jugés essentiels à la question clinique. Tous les articles « clés » retenus par la majorité des membres du Comité ( $\geq 4 / 6$ membres) ont ensuite été examinés par les coprésidents. Les articles se prêtant à un classement [n=16] ont ensuite été soumis à une évaluation critique indépendante. D’autres articles qui fournissent du contexte et des données concernant la question clinique sont cités dans le texte du présent document, mais n'ont pas servi à appuyer les recommandations.

Un groupe externe a examiné la version intégrale des résumés choisis par le Comité d'experts. Ce groupe a procédé à une évaluation critique de chaque document et en a fait rapport au Comité. Leur évaluation a compris le devis et le but de l'étude, le caractère direct de la question de recherche, la qualité méthodologique, les interventions et résultats dignes d'intérêt et l'évaluation des biais potentiels de l'étude. Ils ont également fourni un profil GRADE ${ }^{16}$ des articles examinés à utiliser pour attribuer une cote de preuve. La cote de chaque étude est fondée sur les critères d'attribution de la cote des données probantes ${ }^{17}$ (annexe 3 ) du groupe de travail du profil GRADE. La qualité de la preuve varie de très faible à élevée. Après avoir consulté les responsables de l'évaluation critique, il a été convenu que toutes les études d'observation recevraient le même classement initial « faible » que celui indiqué à l'annexe 3. Les documents très directement liés à la question de recherche ont été utilisés pour élaborer les recommandations. La qualité des preuves à l'appui a servi à déterminer la cote des recommandations (annexe 4) ${ }^{18,19}$. Le Comité d'experts s'est réuni en personne pour examiner les articles évalués d'un point de vue critique et pour formuler des recommandations et leur attribuer une cote. Selon un mandat prédéterminé, il fallait un consensus quant à la formulation et à la notation de chaque recommandation. Les principaux éléments probants des 15 articles qui ont fait l'objet d'une évaluation critique sont résumés à l'annexe 5. (Un article, un examen qualitatif systématique ${ }^{20}$, n'est pas inclus à l'annexe 5 car il ne fournit pas de preuve indépendante.) Les recommandations de ce guide de pratique visent à renforcer et à compléter les normes de pratique actuellement recommandées par le Collège des médecins de famille du Canada et la Société canadienne de pédiatrie (tableau 1).

La version finale du guide de pratique a été approuvée par les organes directeurs pertinents de la Société canadienne d'ophtalmologie, de l'Association canadienne d'ophtalmologie et de strabisme pédiatriques et de l'Association canadienne des optométristes.

ANTÉCÉdENTS NATURELS D’ERREUR DE RÉFRACTION, D’AMBLYOPIE ET DE STRABISME

Les déficiences visuelles affectent 1 à $7 \%$ des enfants selon la définition que l'on en fait ${ }^{21,22}$. Certaines études font même état d'un taux plus élevé de troubles de la vision chez les enfants ${ }^{23}$. Les troubles oculaires les plus fréquents et facilement corrigés sont, de loin, les erreurs de réfraction ${ }^{24-26}$. Les erreurs de réfaction qui produisent une anisométropie sont le facteur de risque le plus courant d'amblyopie.

\section{Erreur de réfraction}

L'erreur de réfraction est un défaut de la capacité de l'œil de focaliser une image avec précision. Les erreurs de réfraction non corrigées peuvent occasionner jusqu'à $69 \%$ des troubles visuels chez les enfant $\mathbf{s}^{27}$. Les erreurs de réfraction entraînent une diminution de la vision du fait que les rayons lumineux de l'image regardée ne convergent pas sur la rétine. Si la longueur axiale de l'œil est trop courte, une hypermétropie se produit. Si elle est trop longue, une myopie se manifeste. Si la puissance de réfraction de l'œil est différente dans un méridien par rapport à un autre, l'astigmatisme en résulte. Selon le degré d'erreur de réfraction et l'âge de l'enfant, les erreurs de réfraction non corrigées peuvent être amblyogènes. 


\section{Amblyopie}

L'amblyopie se définit comme une diminution de la vision, que des lunettes ne corrigent pas, dans un œil autrement sain. La prévalence chez les enfants est estimée à entre 1 et $3 \%$, selon la définition que l'on en fait, et est la cause majeure de perte de vision monoculaire chez les personnes de 20 à 70 ans $^{28-33}$. Les facteurs de risque incluent la prématurité, les troubles neurologiques, les syndromes d'origine génétique et les antécédents familiaux positifs ${ }^{6,22}$. Un diagnostic d'amblyopie est posé quand une différence de deux lignes est constatée entre les yeux et que la correction visuelle est optimale. L'amblyopie bilatérale correspond à une vision pire que 20/40 dans l'œil le moins affecté à l'âge de 4 ans ou moins, ou pire que 20/50 à l'âge de 3 ans ou moins ${ }^{6,33}$. L'œeil opposé peut montrer des déficits subtils ${ }^{34,35}$. L'amblyopie est causée par une carence visuelle de l'œil amblyope durant le développement oculaire - généralement considéré comme s'échelonnant jusqu'à l'âge de dix ans, bien que certaines études proposent un âge plus avancé - qui produit des anomalies structurelles dans le cerveau ${ }^{36}$. (Des études récentes ont remis en question la perte totale de plasticité du cerveau chez les adultes ${ }^{37,38}$, laissant supposer que la vision peut s'améliorer après la période critique du développement oculaire établie par convention ${ }^{39-43}$; cependant, les interventions précoces pourraient toujours produire de meilleurs résultats sur le plan de la vision ${ }^{44}$.) L'amblyopie peut résulter d'une anisométropie, d'un strabisme ou d'une carence occasionnée par l'obstruction de l'axe visuel (p. ex. opacification, obstruction causée par la paupière). Le reste de la voie oculaire et visuelle est normal. Environ $40 \%$ des amblyopes manifestent de l'anisométropie, $40 \%$ un strabisme et $20 \%$ un mécanisme combiné. Une petite proportion présente une obstruction de l'axe visuel ${ }^{45}$. L'anisométropie est une erreur de réfraction inégale dans chaque œil, ce qui entraîne des déformations optiques relatives. L'asymétrie hypermétrope (myopie) est particulièrement amblyogène. L’isométropie élevée (erreur de réfraction égale, mais élevée) peut être amblyogène dans les cas bilatéraux ${ }^{46}$. Un récent rapport regroupant les résultats de deux des plus importantes études sur les maladies oculaires pédiatriques fondées sur la population fournit des estimations du risque d'amblyopie selon divers niveaux d'erreur de réfraction et types de strabisme ${ }^{21}$.

L'amblyopie arrive au deuxième rang des troubles oculaires les plus facilement traitables (après l'erreur de réfraction) lorsqu'elle est décelée et traitée rapidement ${ }^{44,47-50}$. Dans l'ensemble, les bienfaits du dépistage et du traitement, lorsqu'un trouble est décelé, prévalent sur les préjudices et les coûts occasionnés ${ }^{20,51-53}$. En effet, il appert que le traitement de l'amblyopie est l'une des interventions médicales les plus rentables au monde ${ }^{54,55}$. L'amblyopie non ou insuffisamment traitée peut mener à une déficience visuelle et à une réduction de la qualité de vie permanentes. Le traitement échoue dans plus de $20 \%$ des cas ${ }^{45}$ et l'amblyopie peut réapparaitre après le traitement dans jusqu'à $25 \%$ des $\operatorname{cas}^{56,57}$. Un diagnostic précoce peut favoriser la réussite du traitement.

\section{Strabisme}

Le strabisme se produit lorsque les yeux ne sont pas alignés. Il prive le cortex visuel d'une projection simultanée par les zones rétiniennes correspondantes, ce qui entraîne une rivalité et la suppression de la projection par l'œil non dominant. Le résultat est l'amblyopie dans pas moins de $50 \%$ des cas de strabisme ${ }^{58,59}$. Le traitement de l'amblyopie strabique consiste à pénaliser l'œil «bon », bien qu'il existe de nouvelles thérapies comportementales - dont la formation dichoptique et l'apprentissage perceptuel ${ }^{60,61}$ - qui se révèlent prometteuses.

\section{SURVEILLANCE VISUELLE DANS LES SOINS DE PREMIĖRE LIGNE}

Les médecins de famille, les pédiatres généralistes et les autres praticiens de soins de première ligne au Canada utilisent le Rourke Baby Record ${ }^{8}$ ou l'ABCDaire9 (au Québec) pour effectuer leurs examens de routine et l'examen des nourrissons et enfants. Ces deux tests sont fondés sur les meilleures données probantes et font consensus chez les experts. Les éléments particuliers de l'examen de la vue se trouvent au tableau 1. Les recommandations de ce guide visent à renforcer et à compléter ces normes de pratique.

\section{Dépistage}

Les tableaux 1 et $2^{1-9,62}$ présentent un résumé des recommandations sur le dépistage des troubles de la vision au Canada et soulignent le fait que les professionnels de la santé canadiens sont confrontés à des recommandations incohérentes. Dans un sondage réalisé en 2013, on a évalué le respect par les médecins de famille et les pédiatres de l'Ontario des guides de pratique sur le dépistage des troubles de la vision chez les enfants que recommandent la Société canadienne de pédiatrie et le Rourke Baby Record. Sur un total de 3000 questionnaires envoyés par la poste, 719 questionnaires remplis ont été inclus dans l'analyse (taux de réponse de $23,5 \%$ ). À chaque visite régulière d'un enfant, $65 \%$ des médecins de famille et des omnipraticiens et $52 \%$ des pédiatres généraux ont déclaré effectuer un dépistage visuel. Le reflet rétinien a été vérifié par $94 \%$ des médecins chez les enfants de moins de 3 ans, mais seulement par $25 \%$ chez les enfants de plus de 3 ans. Trente-sept pour cent de tous les médecins ont déclaré n'avoir jamais effectué de test d'acuité visuelle dans un groupe d'âge ${ }^{63}$. 
Dans le contexte des soins oculovisuels, le dépistage consiste en un examen sommaire de la fonction visuelle et de l'anatomie oculaire. Le dépistage ne doit pas servir à poser un diagnostic; les patients dont les résultats sont positifs ou à confirmer doivent être dirigés vers un professionnel approprié qui posera un diagnostic et proposera un traitement ${ }^{64}$. Le dépistage de base des troubles de la vision effectué chez les bébés par un médecin de famille ou un pédiatre lors des visites régulières peut permettre de cerner des troubles de la vision traitables en bas âge ${ }^{3}$. Chez les enfants d'âge préscolaire asymptomatiques et à faible risque, il vise davantage le dépistage précoce des troubles prévalents comme l'amblyopie, le strabisme et l'erreur de réfraction non corrigée, ainsi que les troubles plus rares comme les rétinoblastomes et les cataractes congénitales ${ }^{3,62,65}$. Ce dépistage peut être effectué par les pédiatres, les médecins de famille et d'autres fournisseurs de soins de première ligne lors de l'examen régulier des enfants et des nourrissons ${ }^{64,66}$. Le dépistage de l'amblyopie nécessite l'examen des facteurs de risque, lesquels peuvent être diagnostiqués avant l'amblyopie proprement dite (c'est-à-dire avant que la vision formelle ne soit vérifiée). Le dépistage consiste à vérifier la présence d'une erreur de réfraction, d'un strabisme et d'une obstruction de l'axe visuel. Les interventions servant au dépistage du strabisme et de l'amblyopie peuvent inclure les tests d'acuité visuelle, les tests de l'écran (masquage/ démasquage); le dépistage des rétinoblastomes et des cataractes congénitales peut inclure l'examen du reflet rétinien et du fond de l'œeil ${ }^{3,62}$. Les techniques de ces interventions sont décrites dans les écrits ${ }^{3,62,65,67}$. Les patients dont les tests sont positifs doivent être orientés vers un professionnel de la vue qui effectuera des examens plus approfondis.

Les interventions simples de dépistage (examen des antécédents de troubles de la vision dans la famille et toute inquiétude visant un bébé, examen du reflet rétinien, observation du mouvement oculaire, de la pupille et des paupières) sont rapidement et facilement effectuées, mais manquent de sensibilité et de spécificité20,51 . Les tests plus rigoureux nécessitent plus de temps et de ressources, mais présentent une plus grande sensibilité et spécificité20,51-53. L'erreur de réfraction non corrigée est le trouble le plus souvent observé, et le plus simple à corriger, mais elle n'est pas facilement relevée lors des interventions simples de dépistage ${ }^{24-26}$. L'amblyopie, qui affecte 2 à $4 \%$ de la population, peut être soupçonnée par ces interventions, mais nécessite un examen complet avant de proposer un traitement ${ }^{47,53}$. Bien que l'on ne fasse aucune conclusion, il semble que les traitements entrepris le plus tôt possible produisent de meilleurs résultats ${ }^{44,47-50}$.

\section{Examen Complet de la Vue}

Les examens complets de la vue effectués par les optométristes et les ophtalmologistes sont plus élargis et permettent de dépister les troubles oculaires traitables de type amblyogène ou non amblyogène ${ }^{1,3}$. Cela comprend notamment les erreurs de réfraction, le strabisme subtil, les maladies des paupières et des glandes lacrymales et les pathologies rétiniennes s, $1,20,51-53,66$. Ces examens ont une visée diagnostique et mènent à la prise en charge des troubles de l'œil, ce qui comprend notamment l'amblyopie, le strabisme et l'erreur de réfraction non corrigée $\mathrm{e}^{1,2,6,66}$. Les principales composantes d'un examen complet de la vue comprennent l'examen de la réfraction, de l'acuité visuelle, du strabisme, de la vision binoculaire ainsi que de la mobilité et de l'anatomie oculaires (externe et interne) 1,2,6,62,66.

Les techniques d'examen du strabisme et de l'amblyopie dans la population pédiatrique ( 0 à 5 ans) peuvent comprendre l'évaluation de la fixation et de l'acuité visuelle, les tests de l'écran (masquage/démasquage) et du reflet rétinien (méthode de Bruckner), l'examen de reflets cornéens (méthode de Hirschberg), les tests de fusion sensorielle (du filtre rouge, de Worth, etc.), ainsi que les tests de stéréopsie et de motilité oculaire. Les techniques d'examen de la réfraction peuvent comprendre la rétinoscopie (statique ou dynamique), la réfraction manifeste (subjective) et l'autoréfraction (qui n'est généralement pas utilisée dans ce groupe d'âge). Les agents cycloplégiques (gouttes oculaires qui inhibent temporairement l'accommodation) doivent être utilisés conjointement avec ces techniques. Les techniques d'examen supplémentaires comprennent les tests de la pupille, du champ visuel, de la pression intraoculaire, de la vision des couleurs, l'ophtalmoscopie, l'examen avec lampe à fente et l'évaluation externe de la santé oculaire ${ }^{1,6,62,66}$.

\section{EXAMEN DES DONNÉES PROBANTES ET JUSTIFICATION DES RECOMMANDATIONS}

L'objectif principal de la recension des écrits était de trouver des études qui évaluent l'incidence, le cas échéant, du dépistage des troubles de la vision sur la prévalence de l'amblyopie dans l'enfance. Sur les quinze documents clés inclus dans le résumé des constatations (annexe 5), onze ont fourni des données probantes liées à la question de recherche et aux recommandations. Le rendement (qualité ou efficacité) des tests de dépistage menés par les médecins de famille, pédiatres, orthoptistes, optométristes et ophtalmologistes n'a pas été étudié spécifiquement.

Aucun essai clinique randomisé masqué n'a évalué l'efficacité du dépistage des troubles de la vision chez les enfants de 0 à 5 ans; toutefois, des études de cohortes prospectives ont fourni des données probantes cohérentes et robustes selon lesquelles le dépistage des troubles de la vision chez les 8 à 48 mois réduit la prévalence de l'amblyopie à 7 à 
8 ans. Quatre études de trois pays (Israël, Angleterre et Pays-Bas) sont directement liées à notre principale question de recherche et ont été jugées de qualité moyenne dans l'ensemble. L'étude israélienne ${ }^{44}$ est un essai de cohorte prospective mené à Haïfa qui porte sur 808 enfants ayant fait l'objet d'un dépistage entre l'âge de 1 et 2,5 ans et d'un examen de suivi à l'âge de 8 ans (le dépistage comprenait un test de Hirschberg [c.-à-d. le test du réflexe de la lumière cornéenne], un test de masquage et la rétinoscopie sans cycloplégie). Le dépistage de l'amblyopie et de ses facteurs de risque a été effectué par des membres du département d'ophtalmologie du centre médical Bnai-Zion. Les enfants $(\mathrm{n}=782)$ d'une population comparable, mais sans dépistage précoce, ont également été examinés à l'âge de 8 ans. L'amblyopie était 2,6 fois plus susceptible d'être présente dans la cohorte qui n'a pas fait l'objet d'un dépistage (2,6\% $\mathrm{p} / \mathrm{r}$ à $1,0 \%$, respectivement $\mathrm{p}=0,0098$ ). Les enfants qui n'ont pas été soumis à un dépistage avaient aussi une amblyopie plus grave $(1,7 \% \mathrm{p} / \mathrm{r}$ à $0,1 \%$ chez les enfants soumis à un dépistage, $\mathrm{p}=0,00026)$.

Deux études menées en Angleterre par le Avon Longitudinal Study of Parents and Children (ALSPAC), aussi de qualité moyenne, portent sur le dépistage précoce et la prévalence de l'amblyopie ${ }^{48,49}$. Un essai aléatoire imbriqué dans une cohorte prospective a comparé des enfants soumis à de multiples interventions de dépistage orthoptiques entre l'âge de 8 et 37 mois (groupe intensif, $n=2029$ ) et des enfants chez qui une seule intervention de dépistage avait été pratiquée à l'âge de 37 mois (groupe témoin, $n=1490$ ). L'amblyopie était moins prévalente à l'âge de 7,5 ans dans le groupe intensif $(1,45 \% \mathrm{p} / \mathrm{r}$ à $2,66 \%$ dans le groupe témoin, $\mathrm{p}=0,06)$. Cette étude pose un problème important du fait que seulement $54 \%$ des sujets du groupe intensif initial et $55 \%$ de ceux du groupe témoin ont été examinés à lâge de $7,5 \mathrm{ans}^{49}$. La seconde étude, un essai d'observation imbriqué dans la cohorte prospective, a examiné les effets du dépistage orthoptique effectué à l'âge de 4 ou 5 ans par rapport à l'absence de dépistage ${ }^{48}$. La prévalence de l'amblyopie à l'âge de 7,5 ans était inférieure de $45 \%$ chez les enfants d'âge préscolaire ayant fait l'objet d'un test de dépistage par rapport à ceux n'en ayant pas subi $(1,1 \% \mathrm{p} / \mathrm{r}$ à $2,0 \%$, respectivement; $\mathrm{p}=0,052)$. Cette étude n'était pas assez robuste pour montrer des résultats statistiquement significatifs concernant la prévalence de l'amblyopie lorsque les données ont été ajustées pour tenir compte de plusieurs facteurs de confusion potentiellement liés à l'amblyopie.

Des études menées aux Pays-Bas ont également exploré le dépistage précoce et multiple des troubles de la vision dans une seule cohorte de naissance. Les enfants nés à Rotterdam entre septembre 1996 et mai 1997 ont été suivis jusqu'à l'âge de 7 ans. Une étude, dont la qualité a été jugée moyenne dans l'ensemble, a examiné l'effet de multiples dépistages de 1 à 72 mois (2 964 sujets de la cohorte originale de l'étude Rotterdam Amblyopia Screening Effectiveness Study [RAMSES]) sur la prévalence de l'amblyopie à 7 ans. Une prévalence de 3,4 \% de l'amblyopie à l'âge de 7 ans et un effet dose-réponse ont été observés, les enfants ayant subi davantage d'interventions de dépistage montrant des taux plus faibles d'amblyopie ${ }^{47}$. Un autre rapport sur cette même cohorte indique que le dépistage chez les enfants d'âge préscolaire à compter de trois ans contribue le mieux à déceler l'amblyopie ${ }^{51}$. Les auteurs indiquent aussi que l'erreur de réfraction est la cause la plus fréquente d'amblyopie. Aucune de ces études ne portait sur un groupe témoin d'enfants qui n'avaient pas fait l'objet d'un dépistage.

Les études susmentionnées, provenant de différents pays et exposant une variété de tests de dépistage effectués à des âges différents, ont toutes fait état de résultats semblables, soit une prévalence plus faible de l'amblyopie à l'âge de 7 à 8 ans chez les enfants soumis à un dépistage par rapport aux enfants non soumis à un tel test, ainsi que le dépistage multiple par rapport au dépistage unique.

Il a été plus difficile de vérifier les données probantes tirées d'études publiées concernant l'âge optimal pour effectuer un dépistage chez les enfants. D'autres études portant sur l'importance de l'âge au moment du dépistage ont été relevées, mais leur qualité d'ensemble était faible. Un article des Pays-Bas ne cite pas de différence entre le taux de référence à un ophtalmologiste ni la prévalence de l'amblyopie entre une cohorte soumise au dépistage et une autre non soumise au dépistage chez des sujets de 6 à 9 mois $^{68}$. Ce constat peut rendre compte d'un problème relatif aux tests utilisés ou aux examinateurs plutôt qu'à l'âge des enfants. Les références dans les deux groupes étaient fondées principalement sur le strabisme observé et l'on a constaté que de 25 à 50 \% des examinateurs avaient mal effectué les tests de dépistage.

L'effet de la référence précoce en vue du traitement des problèmes d'acuité visuelle et la prévalence de l'amblyopie ont été explorés dans deux autres études prospectives jugées de qualité faible dans l'ensemble. Une étude menée chez des enfants d'Alaska soumis à un programme de dépistage et dirigés vers un spécialiste pour obtenir un traitement a relevé que ces enfants, lorsque traités avant l'âge de 2 ans, ont une meilleure chance d'avoir une acuité visuelle de 6/12; cependant, les résultats de cette étude sont vulnérables à un biais, car plus de $25 \%$ des participants potentiels sont inclus dans l'évaluation du résultat final ${ }^{50}$. Atkinson et ses collègues se sont penchés sur deux programmes de dépistage infantile de Cambridge axés sur les enfants atteints d'hypermétropie ${ }^{53}$. Le premier de 
ces programmes a mené des interventions de dépistage chez 3166 enfants (nés entre 1981 et 1983) à l'âge de 7 et 8 mois et a procédé à un suivi entre les âges de 1 et 3 ans puis à un test d'acuité visuelle à 4 ans. Le deuxième programme a effectué un dépistage chez 5142 enfants (nés entre 1992 et 1994) âgés de 8 mois qui ont eu ensuite jusqu'à onze rendez-vous de suivi jusqu'à l'âge de 7 ans. Ces deux programmes ont signalé une diminution de la prévalence de l'amblyopie chez les enfants atteints d'hypermétropie portant des lunettes en bas âge lorsqu'ils sont évalués à l'âge de 4 ans et de 7 ans, comparativement aux enfants atteints d'hypermétropie ne portant pas de lunettes ${ }^{53}$.

Des trois études transversales qui ont été évaluées, toutes ont reçu des cotes de qualité faibles ou très faibles dans l'ensemble en raison de préoccupations au sujet du biais de sélection potentiel ${ }^{31,26,69}$. Les études jugées de faible qualité provenaient des États-Unis. Une étude du Tennessee portant sur 5548 enfants de 1 à 6 ans relève une très forte prévalence de l'amblyopie chez les enfants atteints d'anisométropie (454/724 ou 62,7 \%) ${ }^{69}$. Donahue indique aussi qu'à l'âge de 3 ans, près des deux tiers des enfants montrant une asymétrie supérieure à 1.0 dioptrie étaient atteints d'amblyopie et que la prévalence de l'amblyopie augmente avec l'âge chez les enfants dont la vision présente une anisométropie. L'étude intitulée Vision in Preschoolers a noté une forte prévalence de l'amblyopie unilatérale (296/3 869 ou 7,7 \%) chez les enfants âgés de 3 à 5 ans inscrits au programme Head Start ${ }^{26}$. Dans ce groupe, le risque accru d'amblyopie a été indépendamment associé à la présence d'un strabisme et d'erreurs de réfraction significatives (notamment la myopie, l'hypermétropie, l'astigmatisme et l'anisométropie).

Une étude transversale australienne a obtenu une cote de qualité d'ensemble médiocre en raison du grand nombre d'enfants en étant exclus parce que la capacité d'effectuer des tests d'acuité visuelle était faible ${ }^{31}$. Cette étude, partie intégrante de l'étude Sydney Paediatric Eye Study, a recruté 2461 enfants ayant entre 6 et 72 mois, mais ses résultats ne portaient que sur 1422 d'entre eux. La prévalence de l'amblyopie était de 27/1 422 (1,9\%) et a été associée de façon significative à l'hypermétropie, à l'astigmatisme, à l'anisométropie et au strabisme ${ }^{31}$.

En résumé, les études prospectives bien menées démontrent clairement qu'à l'âge de 7 ou 8 ans, la prévalence et la gravité de l'amblyopie sont plus faibles dans les cohortes d'enfants soumis à un dépistage en bas âge par rapport aux cohortes non soumises au dépistage ${ }^{44,47-49,51}$. L'âge où le dépistage est effectué dans ces études varie, mais l'on sait, par d'autres études, que plus la détection et le traitement des facteurs de risque de l'amblyopie surviennent tôt, mieux s'en portent les personnes affectées ${ }^{50,53}$. Les facteurs de risque à examiner comprennent les erreurs de réfraction, l'anisométropie et le strabisme $\mathrm{e}^{26,31,69}$.

Sur la base de cette recension, le Comité d'experts a conclu qu'en plus du dépistage de routine effectué par un professionnel de première ligne, un examen complet de la vue réalisé par une personne possédant l'expertise nécessaire pour détecter les facteurs de risque de l'amblyopie - comme un ophtalmologiste ou un optométriste - est recommandé durant la petite enfance. Dans l'ensemble, les résultats corroborent l'importance de la détection précoce de l'amblyopie avant l'âge de 36 mois et au plus tard 48 mois par le dépistage avec au moins un examen complet de la vue avant l'âge de 5 ans.

\section{RECOMMANDATIONS}

- Le dépistage de routine selon l'âge recommandé par Rourke et l'ABCDaire (test du reflet rétinien, de l'écran [masquage/démasquage] et de l'acuité visuelle) chez les nourrissons et les enfants par un fournisseur de soins de première ligne ou un pédiatre devrait se poursuivre ${ }^{8,9}$.

- Si un nourrisson ou un enfant présente une anomalie, il faut le référer au professionnel des soins oculovisuels approprié.

- En plus du dépistage adapté à l'âge, les enfants âgés de 0 à 5 ans devraient faire l'objet d'un examen de la vue par quelqu'un possédant l'expertise nécessaire pour détecter les facteurs de risque d'amblyopie. [1B ${ }^{44,47-49}$ ]

- Idéalement, l'examen de la vue devrait avoir lieu avant l'âge de 3 ans [1B $\left.{ }^{44,47-49}\right]$.

- L'examen de la vue devrait inclure la réfraction et l'évaluation de la motilité oculaire [1B $\left.{ }^{44,47-49}\right]$.

\section{LIMITES}

La principale limite à la mise en œuvre de ce guide de pratique pourrait être l'accès et l'augmentation des ressources nécessaires pour soutenir un tel processus de dépistage. Des efforts supplémentaires devraient donc être consacrés à promouvoir l'accès des enfants à des examens oculovisuels qui détectent les problèmes traitables. 


\section{CONCLUSIONS}

Le dépistage des troubles de la vision effectué par les fournisseurs de soins de première ligne au cours des visites régulières des bébés et des enfants et des vaccinations prévues a été - et continuera d'être - un élément essentiel de la détection des maladies oculaires. L'obtention d'antécédents appropriés lors de l'évaluation du reflet rétinien et de l'examen des annexes externes de l'œil permet de détecter rapidement non seulement les pathologies amblyogènes, mais aussi d'autres maladies potentiellement menaçantes pour la vision (p. ex. cataractes, glaucome) ou mortelles (p. ex., rétinoblastome). Toutefois, le potentiel de détection précoce est limité et un examen oculovisuel complet est également recommandé avant que l'enfant n'entre à l'école. Bien que certains professionnels des soins oculovisuels puissent effectuer des examens complets de la vue dès la naissance en adaptant les techniques, on s'attend à ce que l'enfant puisse, à l'âge de 3 ans, participer à un examen oculovisuel complet. Cela comprend les tests d'acuité visuelle, l'évaluation de la motilité oculaire, l'examen avec lampe à fente, l'examen du fond de l'œil dilaté et la réfraction avec cycloplégie. Si l'amblyopie, le strabisme ou une autre pathologie oculaire est détecté ou soupçonné, et que le problème dépasse le champ de compétences du professionnel qui examine le patient, celui-ci peut être dirigé vers le spécialiste approprié, ce qui permet d'amorcer le traitement en temps opportun.

\section{REMERCIEMENTS}

Cet article est publié simultanément dans le Journal canadien d'ophtalmologie. Le Comité d'experts remercie Cynthia Lank, B.Sc., gestionnaire de projet et directrice de la rédaction, ainsi que Mona Frantzke, M.Sc. L.M., bibliothécaire médicale, pour leur aide. Il remercie aussi les personnes suivantes qui ont réalisé une analyse critique indépendante de la preuve : Ya-Ping Jin, MD PhD, Département d'ophtalmologie et des sciences de la vision, Université de Toronto; Andrei-Alexandru Szigiato, BSc(Hon) MD, chercheur universitaire, glaucome et chirurgie avancée du segment antérieur, Département d'ophtalmologie et des sciences de la vision, Université de Toronto; Alex Lai Chi Tam, M.Sc., Département d'ophtalmologie et des sciences de la vision, Université de Toronto; Sophia Liu, B.Sc. (bioméd.), Faculté de médecine, Université de Toronto.

\section{FINANCEMENT}

Ce projet a été financé conjointement par la Société canadienne d'ophtalmologie et l'Association canadienne des optométristes. Aucun financement de l'industrie n'a été demandé ni fourni.

\section{DIVULGATIONS}

Les membres du comité d'experts ont divulgué tous les conflits et dualités d'intérêts potentiels pour les deux années précédentes (peu importe que ces conflits et dualités aient ou non une incidence sur le guide de pratique).

BER : Aucune affiliation (financière ou autre) à une entreprise commerciale.

WTD : Aucune affiliation (financière ou autre) à une entreprise commerciale.

BT : Aucune affiliation (financière ou autre) à une entreprise commerciale.

JAG : Participation à un essai clinique ayant profité du soutien de Vertex.

LN : Aucune affiliation (financière ou autre) à une entreprise commerciale.

ARL : Aucune affiliation (financière ou autre) à une entreprise commerciale. 
Tableau 1 : Recommandations canadiennes actuelles pour le dépistage des troubles de la vision chez les enfants

\begin{tabular}{|l|l|l|l|l|}
\hline \multicolumn{1}{|c|}{ Organisme } & \multicolumn{1}{|c|}{ SCO } & \multicolumn{1}{|c|}{ ACO } & \multicolumn{1}{c|}{ CMCF } & \multicolumn{1}{c|}{ SCP } \\
\hline $\begin{array}{l}\text { Principales } \\
\text { recommandations }\end{array}$ & s.o. & $\begin{array}{l}\text { Les nourrissons et les tout- } \\
\text { petits doivent subir leur } \\
\text { premier examen de la vue } \\
\text { entre l'âge de 6 et 9 mois; } \\
\text { les enfants d'âge préscolaire } \\
\text { doivent subir au moins un } \\
\text { examen de la vue entre l'âge } \\
\text { de 2 et 5 ans'. }\end{array}$ & $\begin{array}{l}\text { Vérifier le reflet rétinien } \\
\text { pour déceler les maladies } \\
\text { oculaires graves comme } \\
\text { les rétinoblastomes et les } \\
\text { cataractes. } \\
\text { Examen du reflet cornéen } \\
\text { de la lumière / de l'écran et } \\
\text { dépistage du strabisme. } \\
\text { Vérifier l'acuité visuelle } \\
\text { entre l'âge de 3 et 5 ans } s^{8,9} .\end{array}$ & $\begin{array}{l}\text { Vérifier le reflet rétinien } \\
\text { pour déceler les maladies } \\
\text { oculaires graves comme } \\
\text { les rétinoblastomes et les } \\
\text { cataractes. } \\
\text { Examen du reflet cornéen } \\
\text { de la lumière / de l'écran et } \\
\text { dépistage du strabisme. } \\
\text { Vérifier l'acuité visuelle } \\
\text { entre l'âge de 3 et 5 ans. } \\
\text { Les examens oculovisuels } \\
\text { réguliers et complets menés } \\
\text { par des professionnels chez } \\
\text { les enfants en bonne santé } \\
\text { qui ne présentent aucun } \\
\text { facteur de risque n'ont aucun } \\
\text { bienfait prouvé3. }\end{array}$ \\
\hline
\end{tabular}

$\mathrm{ACO}=$ Association canadienne des optométristes, $\mathrm{CMFC}=$ Collège des médecins de famille du Canada, $\mathrm{SCO}=\mathrm{Société}$ canadienne d'ophtalmologie, SCP = Société canadienne de pédiatrie

Tableau 2 : Guides de pratique actuels publiés sur le dépistage des troubles de la vision*

\begin{tabular}{|c|c|c|c|c|c|c|c|c|c|c|}
\hline $\begin{array}{l}\text { Guide } \\
\text { de pra- } \\
\text { tique }\end{array}$ & $\begin{array}{l}0 \text { à } \\
3 \text { mois }\end{array}$ & $\begin{array}{l}3 \text { à } \\
6 \text { mois }\end{array}$ & $\begin{array}{c}6 \text { à } \\
9 \text { mois }\end{array}$ & 3 ans & 2 à 5 ans & $\begin{array}{c}6 \text { à } \\
19 \text { ans }\end{array}$ & $\begin{array}{c}20 \text { à } \\
39 \text { ans }\end{array}$ & $\begin{array}{c}40 \text { à } \\
55 \text { ans }\end{array}$ & $\begin{array}{l}56 \text { à } \\
65 \text { ans }\end{array}$ & $\begin{array}{l}\text { Plus de } \\
65 \text { ans }\end{array}$ \\
\hline $\begin{array}{l}\text { AAP } \\
200362\end{array}$ & & & & Dépistage & & Dépistage & s.o. & & & \\
\hline $\begin{array}{l}\text { AAPOS } \\
20126,7\end{array}$ & \multicolumn{5}{|l|}{ Dépistage } & $\begin{array}{l}\text { Tous } \\
\text { les } 1 \text { ou } \\
2 \text { ans }\end{array}$ & \multicolumn{4}{|l|}{ s.o. } \\
\hline $\begin{array}{l}\text { ACMTS } \\
20074\end{array}$ & \multicolumn{4}{|c|}{$\begin{array}{l}\text { Les programmes préscolaires de dépistage des } \\
\text { troubles de la vision variaient d'une province à } \\
\text { l'autre, qu'il s'agisse des examens effectués par } \\
\text { le personnel infirmier en santé publique ou des } \\
\text { examens d'optométrie complets; aucun n'est } \\
\text { supérieur. }\end{array}$} & Dépistage & \multicolumn{5}{|l|}{ s.o. } \\
\hline $\begin{array}{l}\text { ACO } \\
20121\end{array}$ & & & $\begin{array}{l}\text { Examen } \\
\text { de la vue }\end{array}$ & & $\begin{array}{l}\text { Examen } \\
\text { de la vue }\end{array}$ & $\begin{array}{l}\text { Chaque } \\
\text { année }\end{array}$ & $\begin{array}{l}\text { Tous les } 2 \\
\text { ou } 3 \text { ans }\end{array}$ & $\begin{array}{l}\text { Tous les } \\
2 \text { ans }\end{array}$ & $\begin{array}{l}\text { Tous les } \\
2 \text { ans }\end{array}$ & $\begin{array}{l}\text { Chaque } \\
\text { année }\end{array}$ \\
\hline $\begin{array}{l}\text { SCO } \\
20072\end{array}$ & \multicolumn{6}{|l|}{ s.o. } & $\begin{array}{l}\text { Au moins } \\
\text { tous les } \\
10 \text { ans }\end{array}$ & $\begin{array}{l}\text { Au } \\
\text { moins } \\
\text { tous les } \\
5 \text { ans }\end{array}$ & $\begin{array}{l}\mathrm{Au} \\
\text { moins } \\
\text { tous les } \\
3 \text { ans }\end{array}$ & $\begin{array}{l}\mathrm{Au} \\
\text { moins } \\
\text { tous les } \\
2 \text { ans }\end{array}$ \\
\hline $\begin{array}{l}\text { SCP } \\
20093\end{array}$ & Dépistage & & Dépistage & & Dépistage & Dépistage & \multicolumn{4}{|l|}{ s.o. } \\
\hline $\begin{array}{l}\text { USPSTF } \\
20175\end{array}$ & & Dépistage & & & Dépistage & \multicolumn{5}{|l|}{ s.o. } \\
\hline
\end{tabular}

* Veuillez consulter les documents originaux pour obtenir tous les détails. Ce tableau met en évidence les différences dans la portée et les recommandations des divers guides de pratique sans résumer complètement chaque document. $\mathrm{AAP}=$ American Academy of Pediatrics, AAPOS = American Association for Pediatric Ophthalmology and Strabismus, ACMTS = Agence canadienne des médicaments et des technologies de la santé, ACO = Association canadienne des optométristes, $\mathrm{SCO}=$ Société canadienne d'ophtalmologie, $\mathrm{SCP}=$ Société canadienne de pédiatrie, s.o. = Ces guides de pratique ne traitent pas de ces groupes d'âge, USPSTF = United States Preventive Services Task Force 
ANNEXE 1 : Recherche dans Medline et Embase

\section{Test de l'indice : (1)}

Vision Tests[MeSH:noexp] OU Diagnostic Techniques, Ophthalmological[MAJR:noexp] OU ((Refraction, Ocular[MAJR] OU Visual Acuity[MAJR]) ET (exam*[tw] OU test[tw] OU tests[tw] OU assessment*[tw]))

\section{Dépistage indiciel : (2)}

Vision Screening[MeSH] OU ((screening[tw] OU Mass Screening[MeSH]) ET (eye[tw] OU vision[tw] OU ocular[tw] OU visual[tw] OU ophthalmic[tw]))

\section{Condition cible : (3)}

Amblyopia[MeSH] OU amblyopia[tw] OU Strabismus[MeSH] OU strabismus[tw] OU Refractive Errors[Mesh] OU refractive-error*[tw] OU refractive-disorder*[tw] OU lazy-eye*[tw] OU squint[tw] OU cross-eye*[tw] OU astigmatism $[\mathrm{tw}]$ OU presbyopia[tw] OU myopia[tw] OU hyperopia[tw] OU anisometropia[tw] OU ocularalignment[tw] OU Vision Disorders/diagnosis[MAJR:noexp] OU Eye Diseases/diagnosis[MAJR:noexp]

Mots-clés applicables au contexte : (4)

Evidence-Based Practice[MeSH] OU evidence-based[tw] OU Early Diagnosis[MeSH:noexp] OU early-diagnosis[tw] OU early-diagnostic $[\mathrm{tw}]$ OU undetected[tw] OU uncorrected[tw] OU visual-impairment[tw] OU "Referral and Consultation"[MeSH] OU Early Medical Intervention[MeSH] OU Risk Factors[MeSH] OU Age of Onset[MeSH] OU Time Factors[MeSH] OU Advisory Committees[MeSH] OU guideline[pt] OU practice-guideline[pt] OU "Consensus Development Conference" [pt] OU guideline*[tw] OU consensus[tw] OU recommendation*[tw] OU Ophthalmology[MeSH:noexp] OU Optometry[MeSH:noexp] OU optometrist*[tw] OU ophthalmologist*[tw] OU pediatrician[tw] OU paediatrician[tw] OU Primary Health Care[MeSH] OU ((comprehensive[tw] OU routine[tw] OU periodic [tw] OU population-based[tw] OU whole-population[tw] OU universal[tw] OU gold-standard[tw] OU Asymptomatic Diseases[MeSH] OU asymptomatic[tw] OU schedule[tw]) ET (eye[tw] OU vision[tw] OU ocular[tw] OU visual[tw] OU ophthalmic [tw]))

\section{Groupe d'âge cible : (5)}

Child, Preschool[MeSH] OU Infant[MeSH] OU preschool[tw] OU pre-school[tw] OU kindergarten[tw] OU kindergarden[tw] OU Pediatrics[MeSH] OU pediatric*[tw] OU paediatric*[tw] OU children[tw]

\section{Limites : (6)}

(English[lang] OU French[lang]) ET («1995/01/01»[PDAT]: «3000/12/31»[PDAT])

\section{Dernière recherche sur Medline}

(1 OU 2) ET (3 OU 4) ET 5 ET 6

La recherche effectuée dans Embase est identique à celle effectuée dans Medline, sans les termes MeSH toutefois et excluant les fichiers Medline.

ANNEXE 2 : Stratégie de recension des écrits : critères d'inclusion et d'exclusion

Critères d'inclusion : Études portant sur des enfants ayant subi des interventions entre la naissance et l'âge de 5 ans; essais cliniques bien menés et études d'observation; études sur l'amblyopie, les facteurs de risque de l'amblyopie et l'erreur de réfraction; articles de recherche publiés dans des périodiques à comité de sélection rédigés en anglais ou en français; études menées dans des établissements de première ligne et les infrastructures fondées sur la population; études portant sur les examens de dépistage habituellement offerts dans les établissements de soins de première ligne (examen de l'acuité visuelle et du reflet rétinien et test de l'écran, par exemple) ou techniques d'examen utilisées par les optométristes et les ophtalmologistes (rétinoscopie, etc.); études portant sur les résultats suivants : amélioration de l'acuité visuelle, diminution de l'amblyopie, meilleur rendement scolaire et meilleure qualité de vie.

Critères d'exclusion : Études portant sur des enfants de 6 ans ou plus; articles sur des complications oculaires causées par d'autres maladies (comme le diabète); articles sur des sous-groupes de patients atteints de maladies oculaires diagnostiquées (comme le glaucome, les rétinopathies des prématurés, la dégénérescence maculaire liée à l'âge ou les troubles oculaires causés par le diabète); articles non axés sur les résultats en matière de vision; articles évaluant l'utilité ou la rentabilité d'outils numériques ou d'instruments de dépistage particuliers (comme la téléophtalmologie, les appareils de dépistage portatifs, les appareils de dépistage numériques, l'auto-réfractomètre Retinomax); articles évaluant les programmes de dépistage (comme ceux dans les milieux scolaires ou les établissements de soins prolongés); articles portant sur les traitements ou sur le respect des traitements par les patients; articles issus de pays dont la composition ethnique ou le système de santé diffèrent grandement de ceux du Canada; articles décrivant des programmes existants; articles décrivant des politiques territoriales; articles d'opinion et éditoriaux; examen de dossiers; articles rédigés dans une langue autre que l'anglais ou le français; articles sur la prévention de la perte de vision; articles s'adressant au personnel infirmier en milieu scolaire et aux orthoptistes; documents de politique; articles sur les ressources et la main-d'œuvre en santé; articles sur l'adoption de recommandations de lignes directrices; articles sur des groupes de discussion et des données de sondage et articles jugés obsolètes. 
ANNEXE 3 : Critères d'attribution de la cote de preuve (selon les critères GRADE) ${ }^{17}$

\begin{tabular}{|l|l|}
\hline \multirow{2}{*}{ Types de preuve } & $\begin{array}{l}\text { - Essai aléatoire = élevé } \\
\text { - Étude d'observation = faible } \\
\text { - Tout autre élément de preuve = très faible }\end{array}$ \\
\hline Diminuer* la cote si... & $\begin{array}{l}\text { - Limites graves ou très graves à la qualité de l'étude } \\
\text { - Importante incohérence } \\
\text { - Certaine ou grande incertitude quant au lien direct avec la question de recherche } \\
\text { - Données imprécises ou rares } \\
\text { - Forte probabilité de déceler un biais }\end{array}$ \\
\hline Augmenter la cote si... & $\begin{array}{l}\text { - Forte preuve d'association - risque relatif important }>2(<0,5) \text { d'après des données probantes } \\
\text { cohérentes provenant de deux études d'observation ou plus, sans facteurs de confusion plausibles }(+1) \\
\text { - sans aucune menace majeure à la validité }(+2)\end{array}$ \\
\hline \multirow{2}{*}{ Portée } & - Tous les facteurs de confusion plausibles ayant réduit l'effet $(+1)$
\end{tabular}

* Chaque critère de qualité peut réduire la qualité de 1 ou, s’il est très sérieux, de 2 niveaux.

APPENDIX 4: Classement des recommandations en fonction de la force de la recommandation (1-2), des conséquences et de la qualité de la preuve (confiance dans l'estimation de l'effet, $A-C$ ); d'après les directives GRADE $E^{18,19}$

\begin{tabular}{|c|c|c|}
\hline $\begin{array}{l}\text { Niveau de recommandation } \\
\text { (Implication) }\end{array}$ & Estimation de l'effet & Qualité des preuves \\
\hline $\begin{array}{l}\text { 1A : Recommandation forte, } \\
\text { données probantes de } \\
\text { grande qualité } \\
\text { (s'applique à la plupart des } \\
\text { patients) }\end{array}$ & $\begin{array}{l}\text { Preuves très solides de risque } \\
\text { relatif important. }\end{array}$ & $\begin{array}{l}\text { Preuves provenant de plus d'un essai contrôlé aléatoire } \\
\text { bien exécuté ou preuves solides sous une autre forme. } \\
\text { Il est peu probable que d'autres recherches modifient la } \\
\text { confiance dans les estimations de l'effet. }\end{array}$ \\
\hline $\begin{array}{l}\text { 1B : Recommandation forte, } \\
\text { données probantes de } \\
\text { qualité moyenne } \\
\text { (s'applique à la plupart des } \\
\text { patients) }\end{array}$ & $\begin{array}{l}\text { Preuves solides d'un risque relatif } \\
\text { important. }\end{array}$ & $\begin{array}{l}\text { Preuves provenant d'essais cliniques aléatoires } \\
\text { montrant des limites importantes (résultats } \\
\text { incohérents, défauts méthodologiques ou imprécision) } \\
\text { ou preuves très solides de certains autres devis de } \\
\text { recherche. D'autres recherches (si elles sont effectuées) } \\
\text { pourraient modifier l'estimation de l'effet. }\end{array}$ \\
\hline $\begin{array}{l}\text { 1C : Recommandation forte, } \\
\text { données probantes de faible } \\
\text { qualité } \\
\text { (s'applique à la plupart des } \\
\text { patients) }\end{array}$ & $\begin{array}{l}\text { Les avantages semblent surpasser } \\
\text { les risques et les fardeaux, ou vice } \\
\text { versa. }\end{array}$ & $\begin{array}{l}\text { Preuves tirées d'études d'observation, d'expériences } \\
\text { cliniques non systématiques ou d'essais cliniques } \\
\text { aléatoires comportant de graves lacunes. D'autres } \\
\text { recherches sont susceptibles de modifier l'estimation } \\
\text { de l'effet. }\end{array}$ \\
\hline $\begin{array}{l}2 \mathrm{~A}: \text { Recommandation } \\
\text { faible, données probantes de } \\
\text { grande qualité } \\
\text { (ne s'applique pas à tous les } \\
\text { patients) }\end{array}$ & $\begin{array}{l}\text { Avantages faisant réellement } \\
\text { contrepoids aux risques et aux } \\
\text { fardeaux. }\end{array}$ & $\begin{array}{l}\text { Preuves provenant de plus d'un essai clinique aléatoire } \\
\text { bien exécuté, ou preuves solides d'une autre forme. Il } \\
\text { est peu probable que d'autres recherches modifient la } \\
\text { confiance dans l'estimation de l'effet. }\end{array}$ \\
\hline $\begin{array}{l}\text { 2B : Recommandation } \\
\text { faible, données probantes de } \\
\text { qualité moyenne } \\
\text { (d'autres approches } \\
\text { pourraient faire mieux) }\end{array}$ & $\begin{array}{l}\text { Avantages faisant réellement } \\
\text { contrepoids aux risques et aux } \\
\text { fardeaux, mais laissant planer } \\
\text { une certaine incertitude dans } \\
\text { l'estimation des avantages, des } \\
\text { risques et des fardeaux. }\end{array}$ & $\begin{array}{l}\text { Preuves provenant d'essais cliniques aléatoires } \\
\text { montrant des limites importantes (résultats } \\
\text { incohérents, défauts méthodologiques ou imprécision) } \\
\text { ou preuves très solides de certains autres devis de } \\
\text { recherche. D'autres recherches (si elles sont effectuées) } \\
\text { pourraient modifier l'estimation de l'effet. }\end{array}$ \\
\hline $\begin{array}{l}2 \mathrm{C}: \text { Recommandation faible, } \\
\text { données probantes de piètre } \\
\text { qualité } \\
\text { (d'autres approches } \\
\text { pourraient faire mieux) }\end{array}$ & $\begin{array}{l}\text { Incertitude par rapport à l'estimation } \\
\text { des avantages, des risques et des } \\
\text { fardeaux; les avantages pourraient } \\
\text { faire contrepoids aux risques et aux } \\
\text { fardeaux. }\end{array}$ & $\begin{array}{l}\text { Preuves tirées d'études d'observation, d'expériences } \\
\text { cliniques non systématiques ou d'essais cliniques } \\
\text { aléatoires comportant de graves lacunes. D'autres } \\
\text { recherches sont susceptibles de modifier l'estimation } \\
\text { de l'effet. }\end{array}$ \\
\hline
\end{tabular}




\begin{tabular}{|c|c|c|c|c|}
\hline \multirow{7}{*}{ 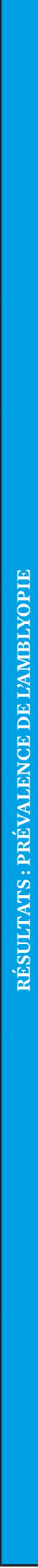 } & 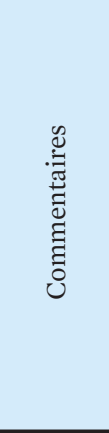 & 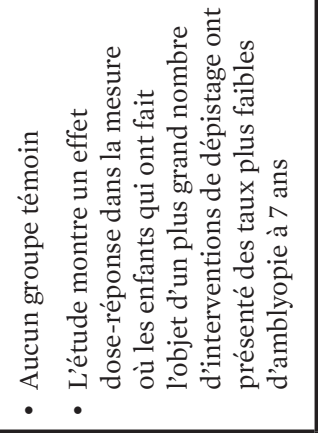 & 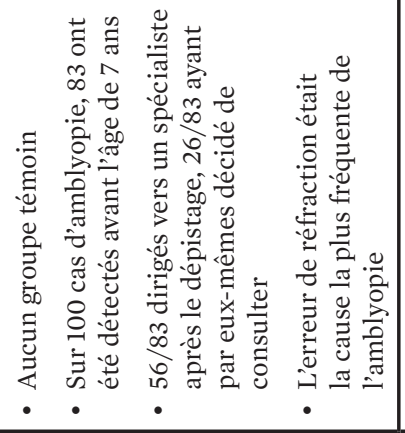 & 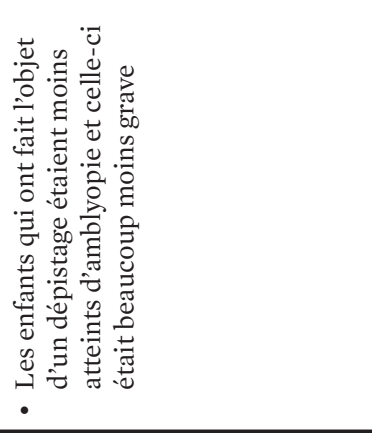 \\
\hline & 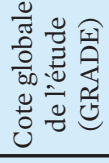 & 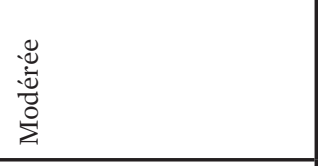 & 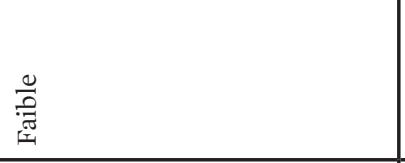 & 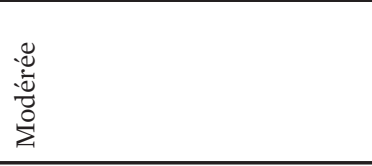 \\
\hline & 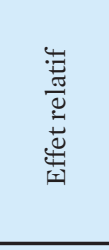 & 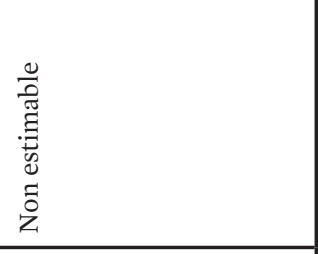 & 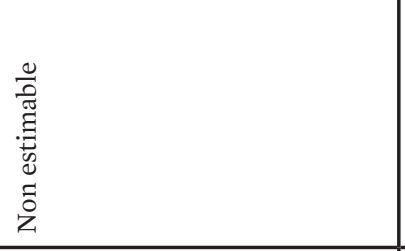 & 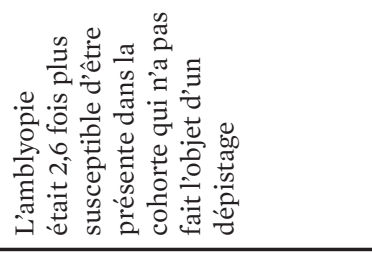 \\
\hline & 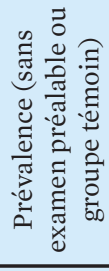 & 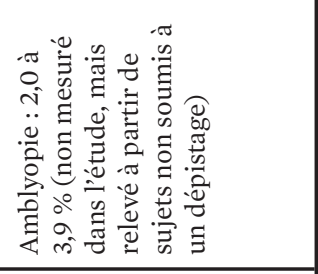 & & 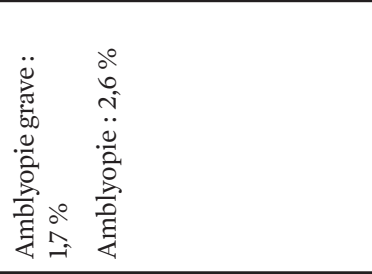 \\
\hline & 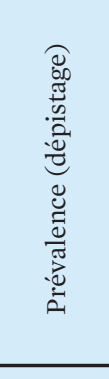 & 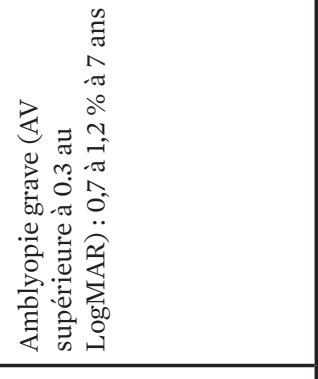 & 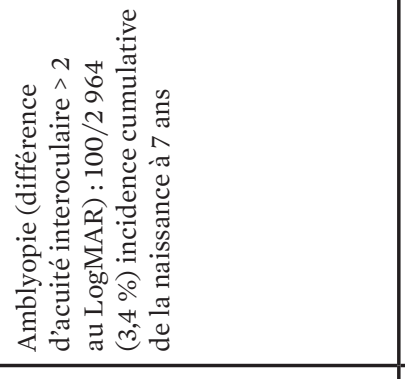 & 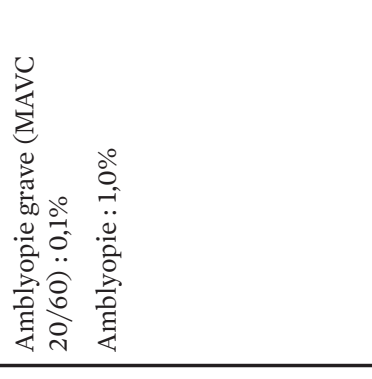 \\
\hline & 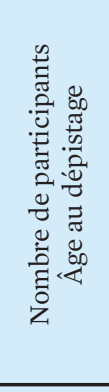 & 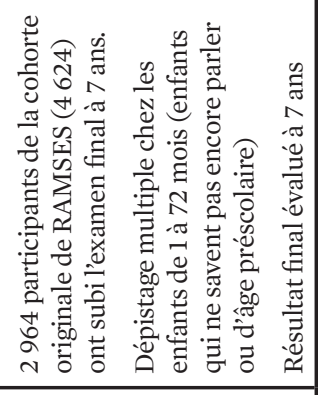 & 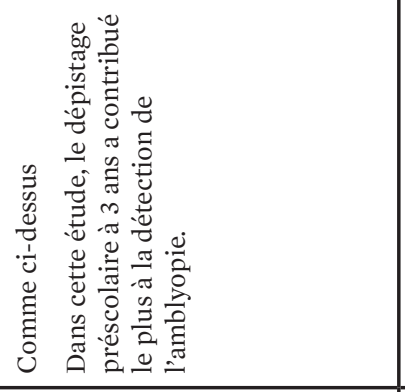 & 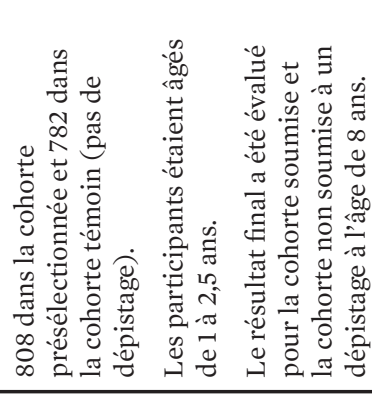 \\
\hline & 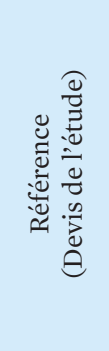 & 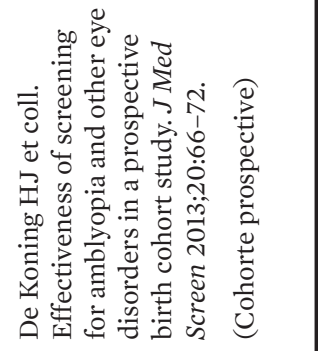 & 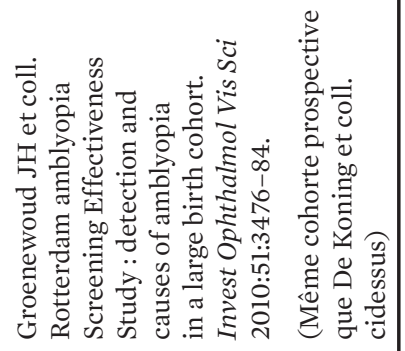 & 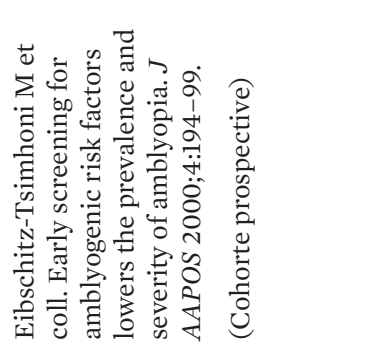 \\
\hline
\end{tabular}




\begin{tabular}{|c|c|c|c|c|c|c|c|}
\hline 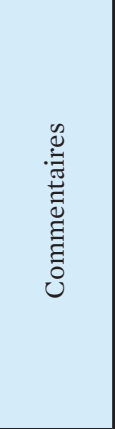 & 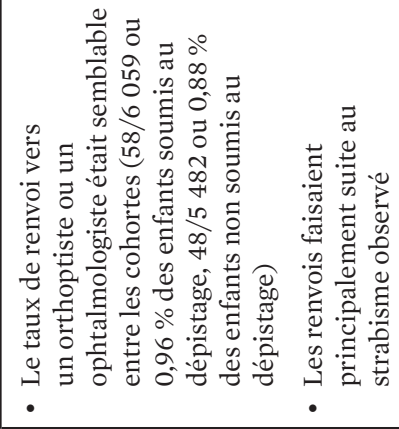 & 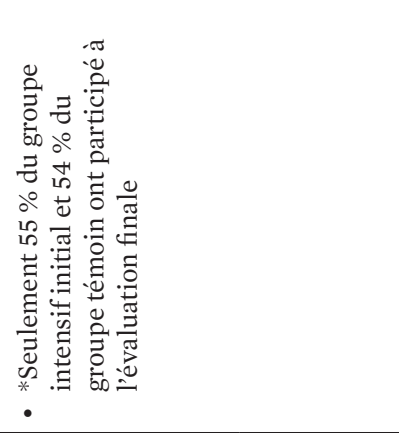 & & 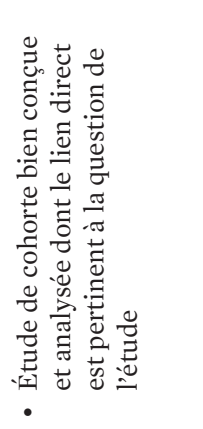 & & & \\
\hline 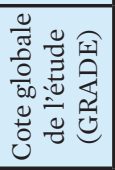 & 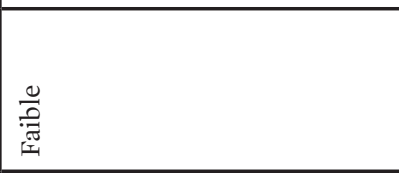 & 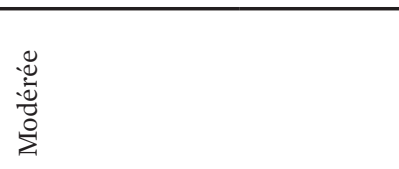 & & 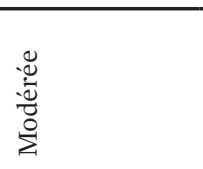 & & & \\
\hline 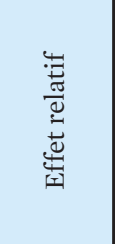 & & 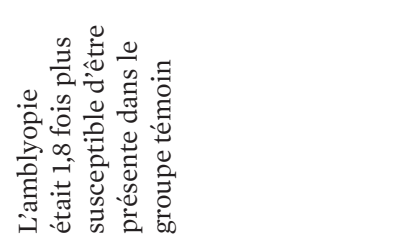 & & 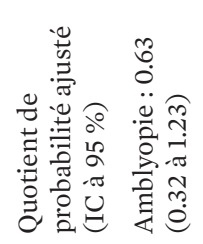 & 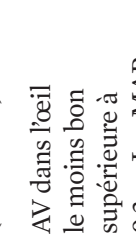 & 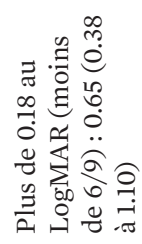 & \\
\hline 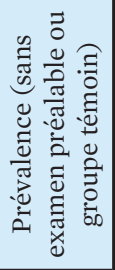 & 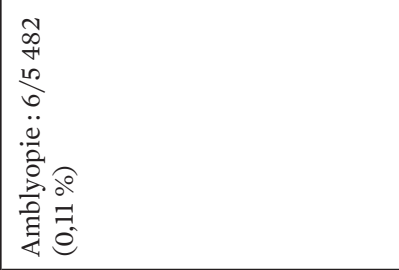 & 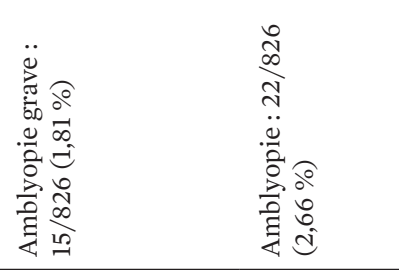 & & 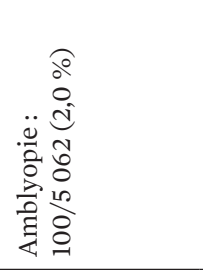 & 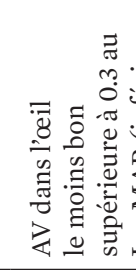 & 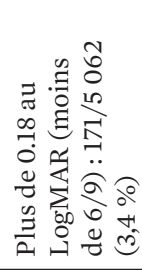 & \\
\hline 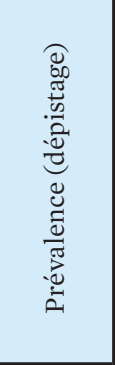 & 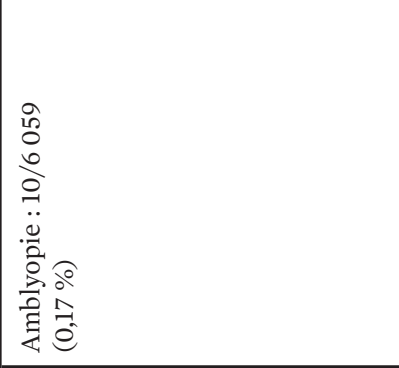 & 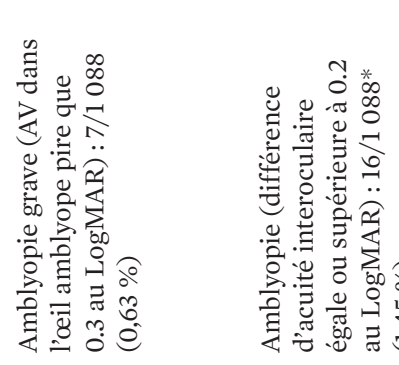 & & 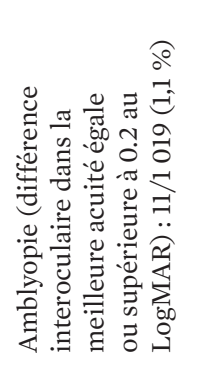 & 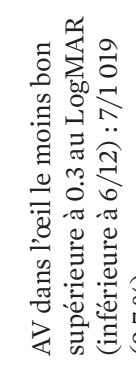 & 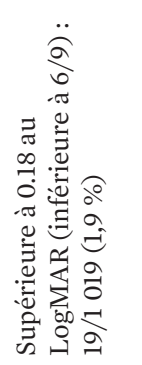 & 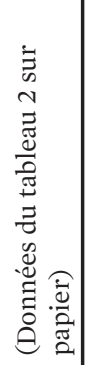 \\
\hline 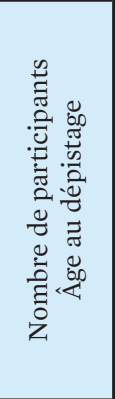 & 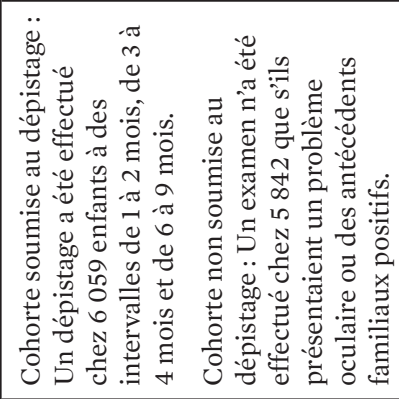 & 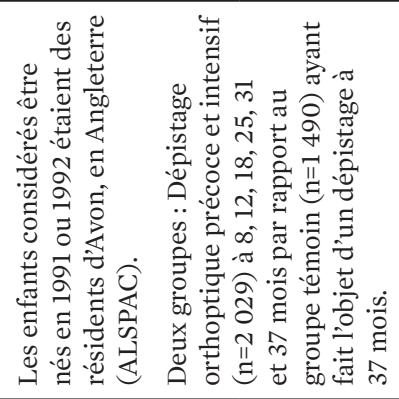 & 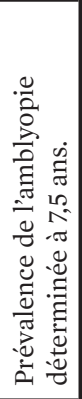 & 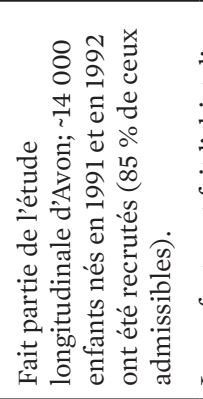 & 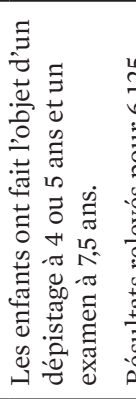 & & \\
\hline 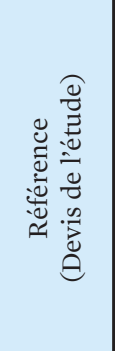 & 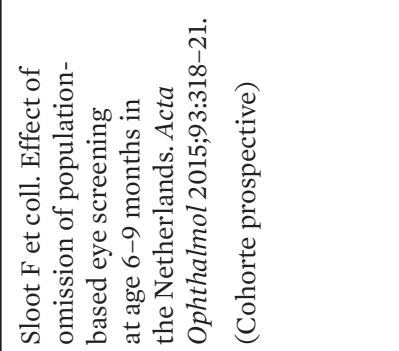 & 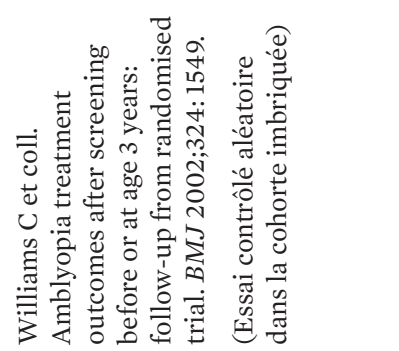 & & 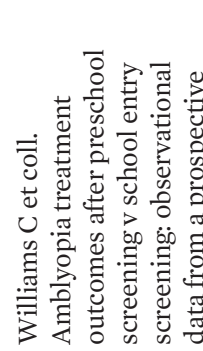 & 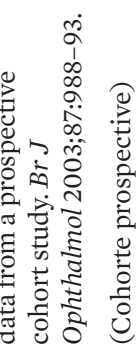 & & \\
\hline
\end{tabular}




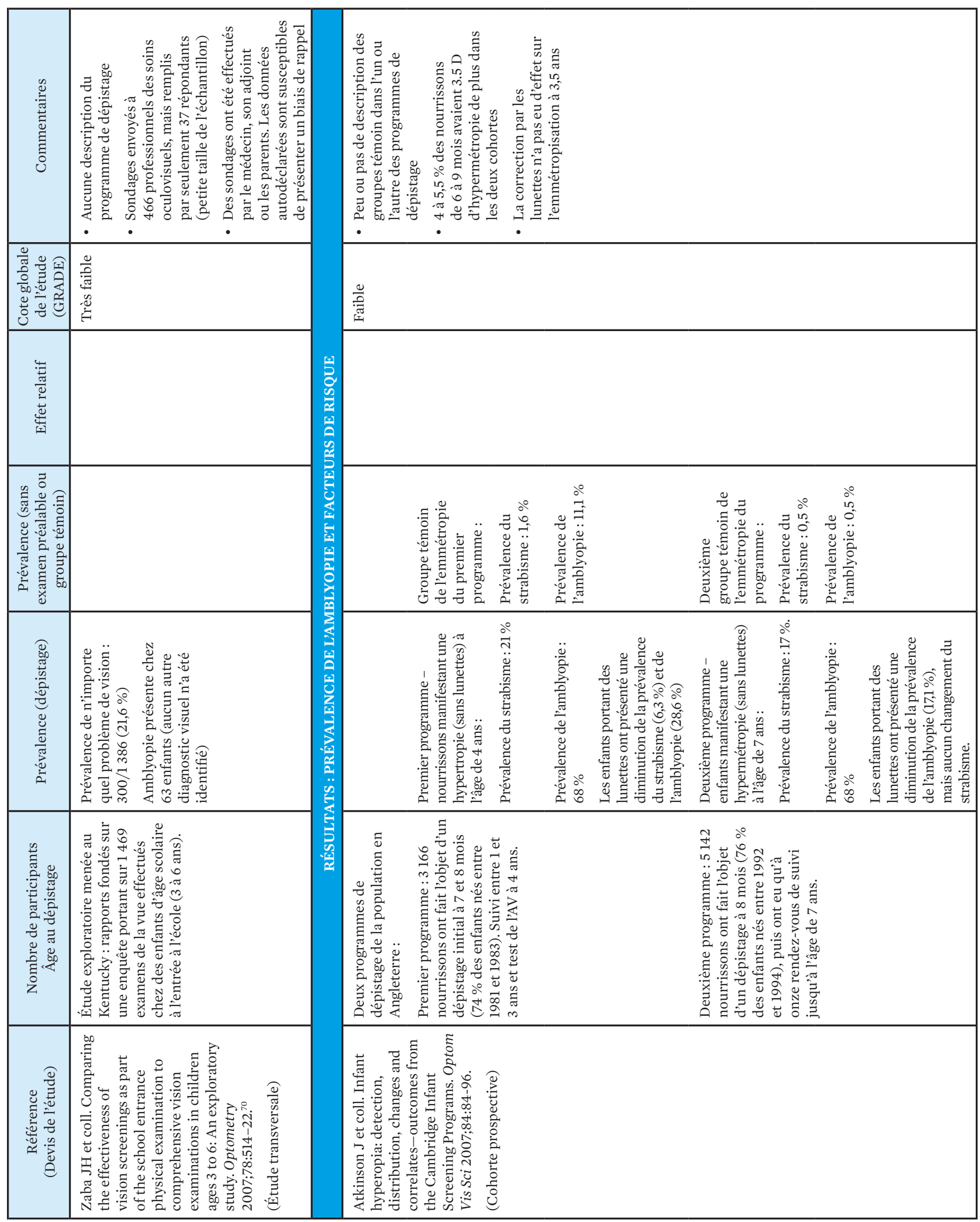




\begin{tabular}{|c|c|c|}
\hline 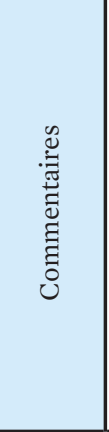 & 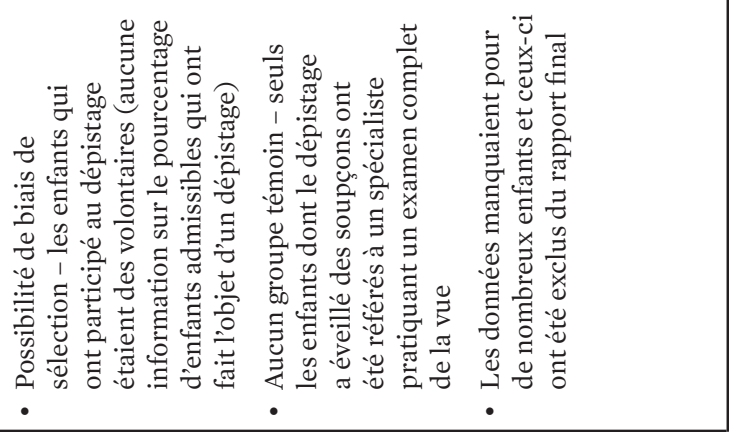 & 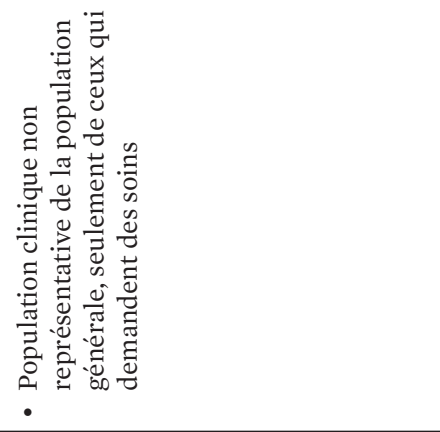 \\
\hline 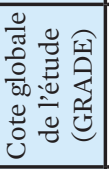 & 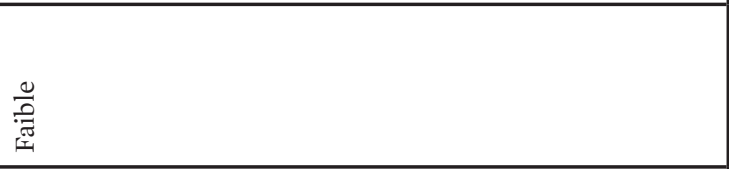 & 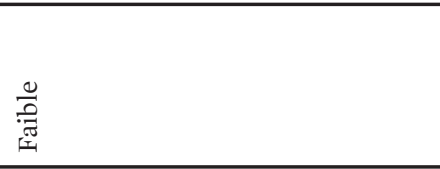 \\
\hline 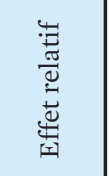 & & \\
\hline 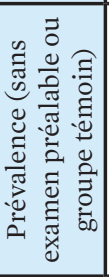 & & \\
\hline 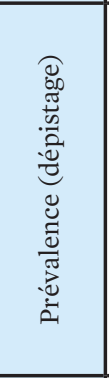 & 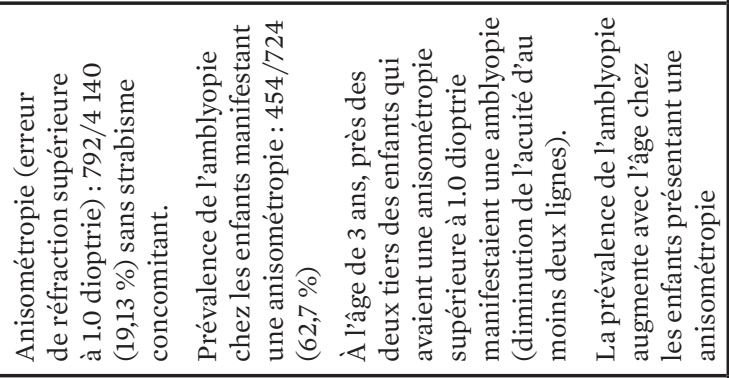 & 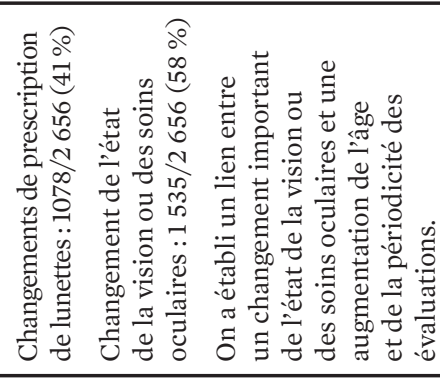 \\
\hline 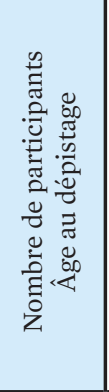 & 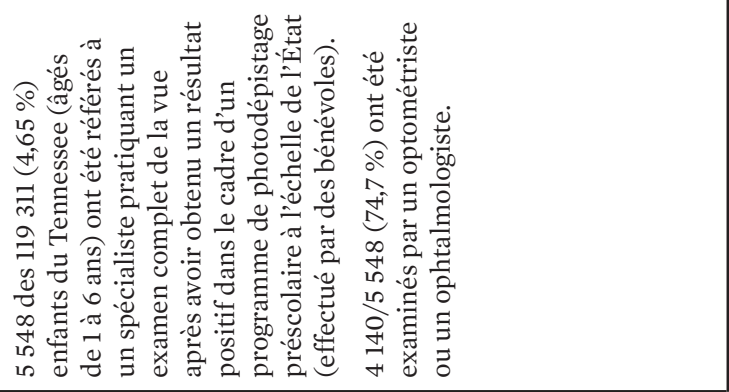 & 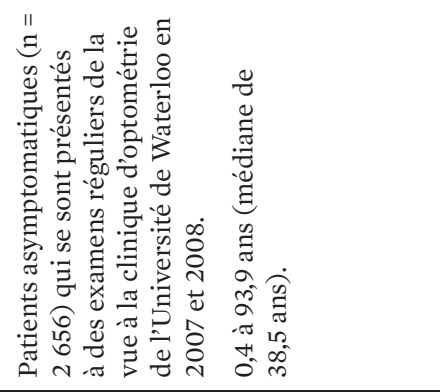 \\
\hline 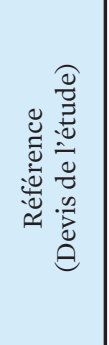 & 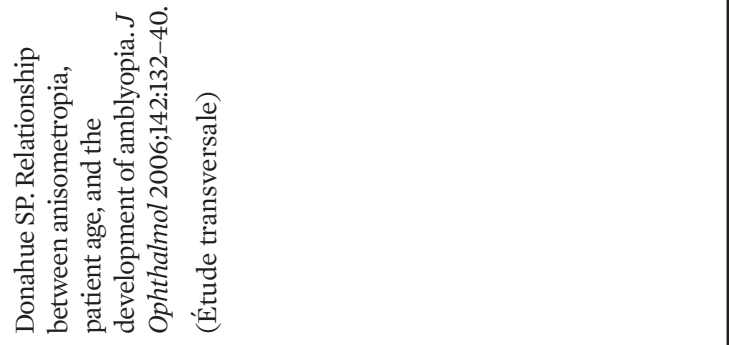 & 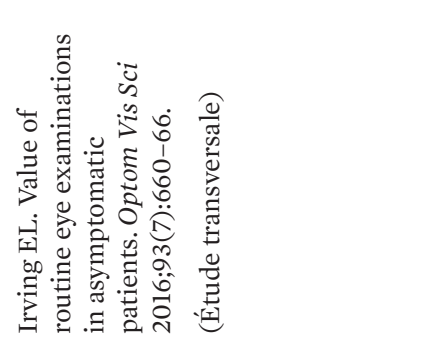 \\
\hline
\end{tabular}




\begin{tabular}{|c|c|c|}
\hline 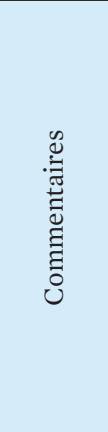 & 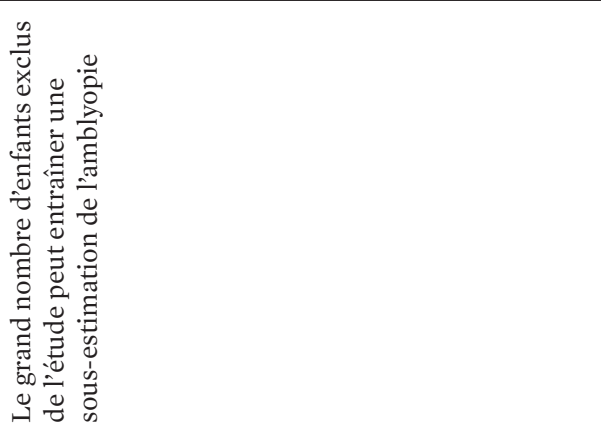 & 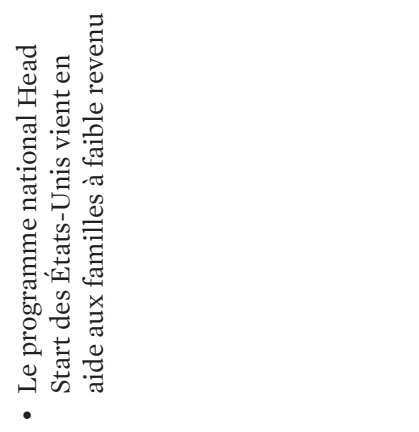 \\
\hline 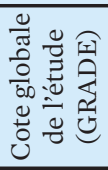 & & $\begin{array}{l}\frac{0}{7} \\
\frac{\pi}{\pi} \\
\end{array}$ \\
\hline 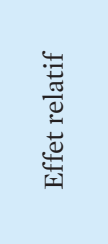 & 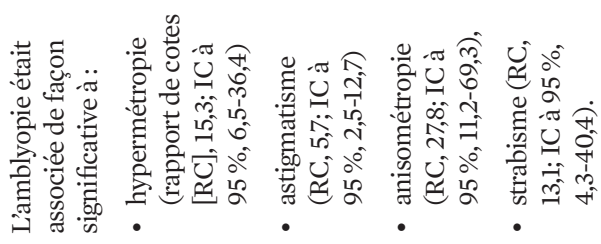 & 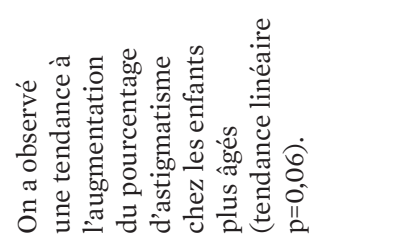 \\
\hline 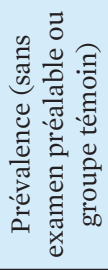 & & \\
\hline 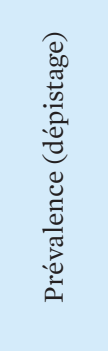 & 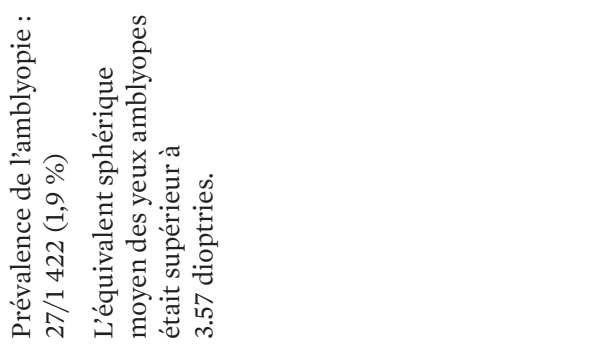 & 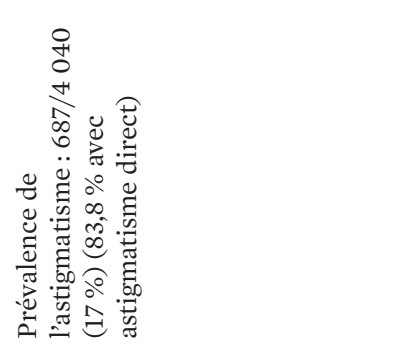 \\
\hline 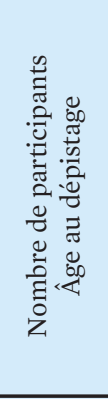 & 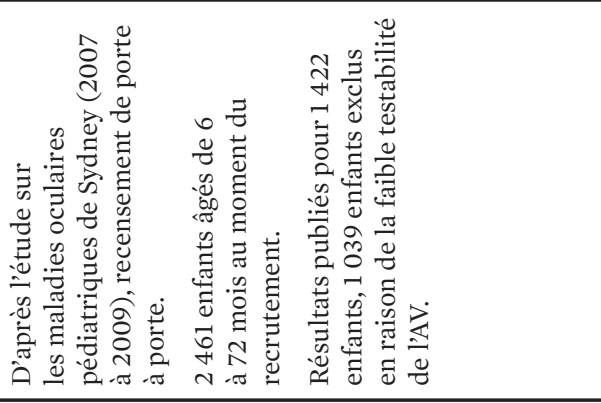 & 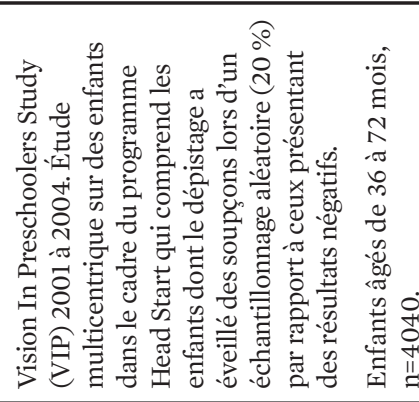 \\
\hline 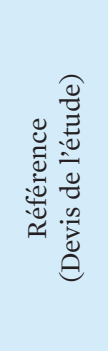 & 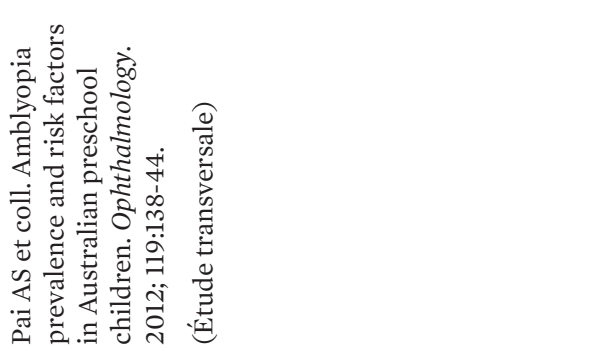 & 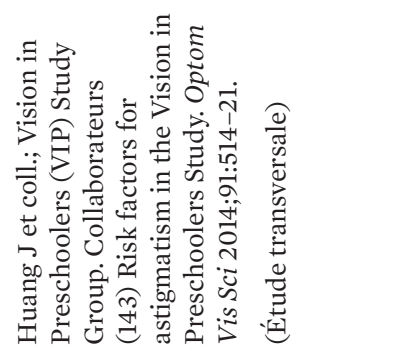 \\
\hline
\end{tabular}




\begin{tabular}{|c|c|c|}
\hline 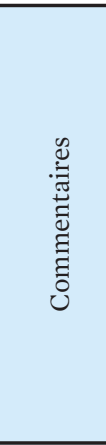 & 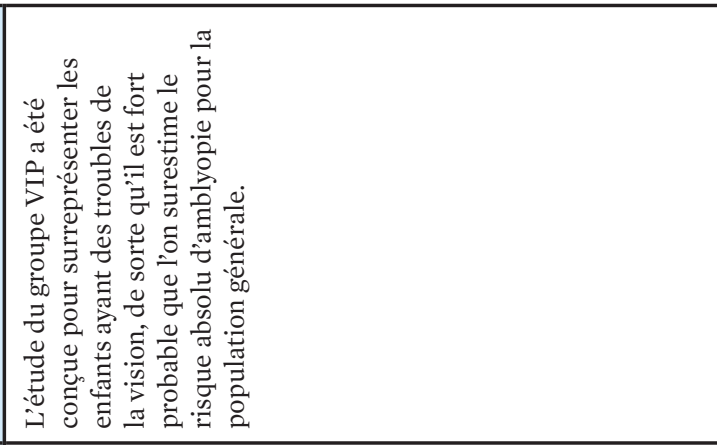 & 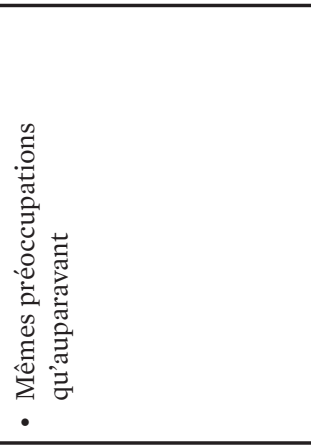 \\
\hline 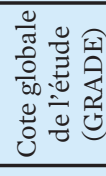 & 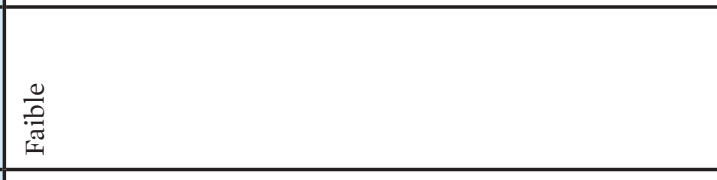 & 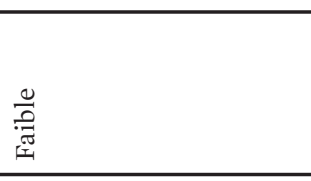 \\
\hline 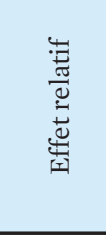 & 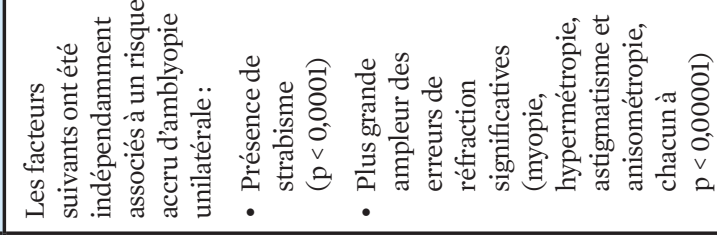 & \\
\hline 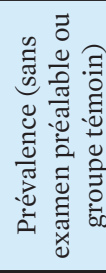 & & \\
\hline 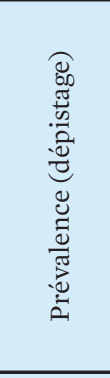 & 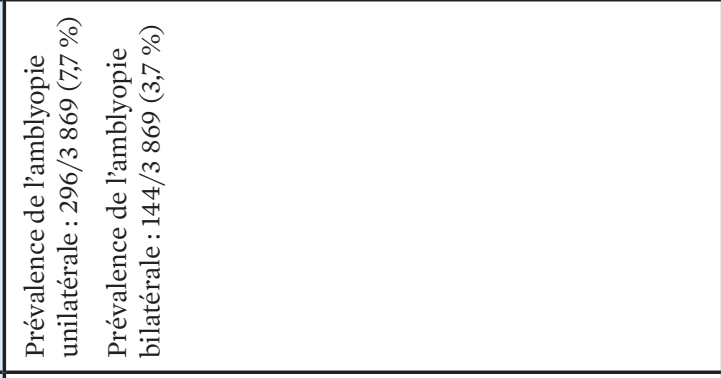 & 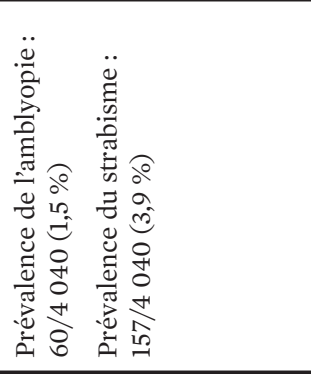 \\
\hline 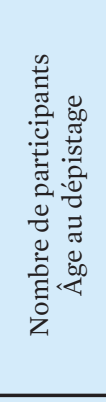 & 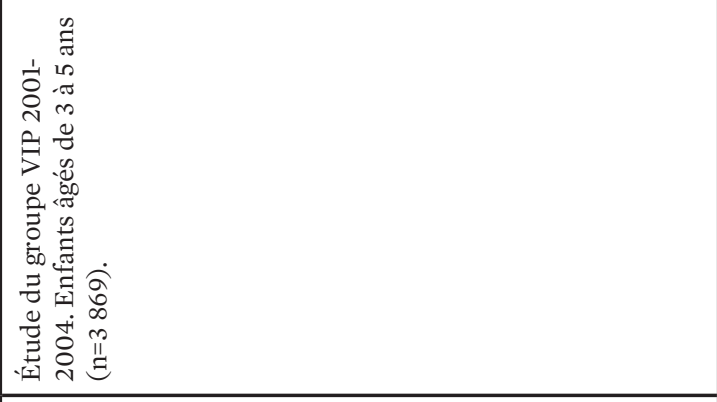 & 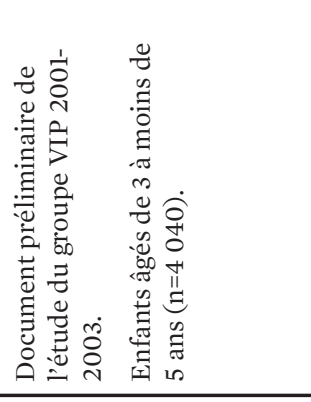 \\
\hline 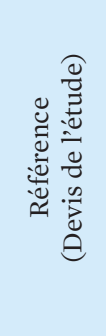 & 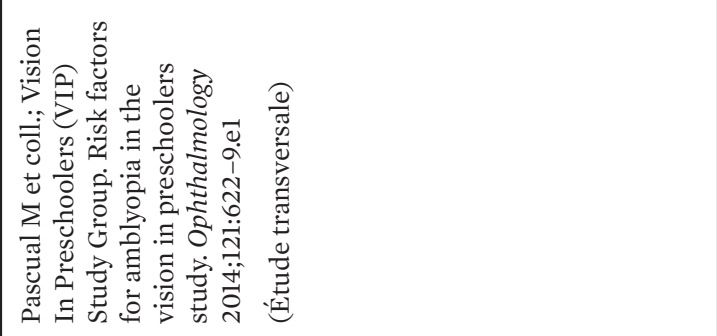 & 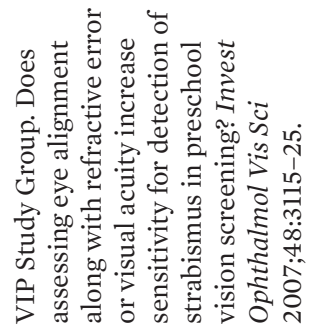 \\
\hline
\end{tabular}


C LIGNES DIRECTRICES DE PRATIQUE CLINIQUE

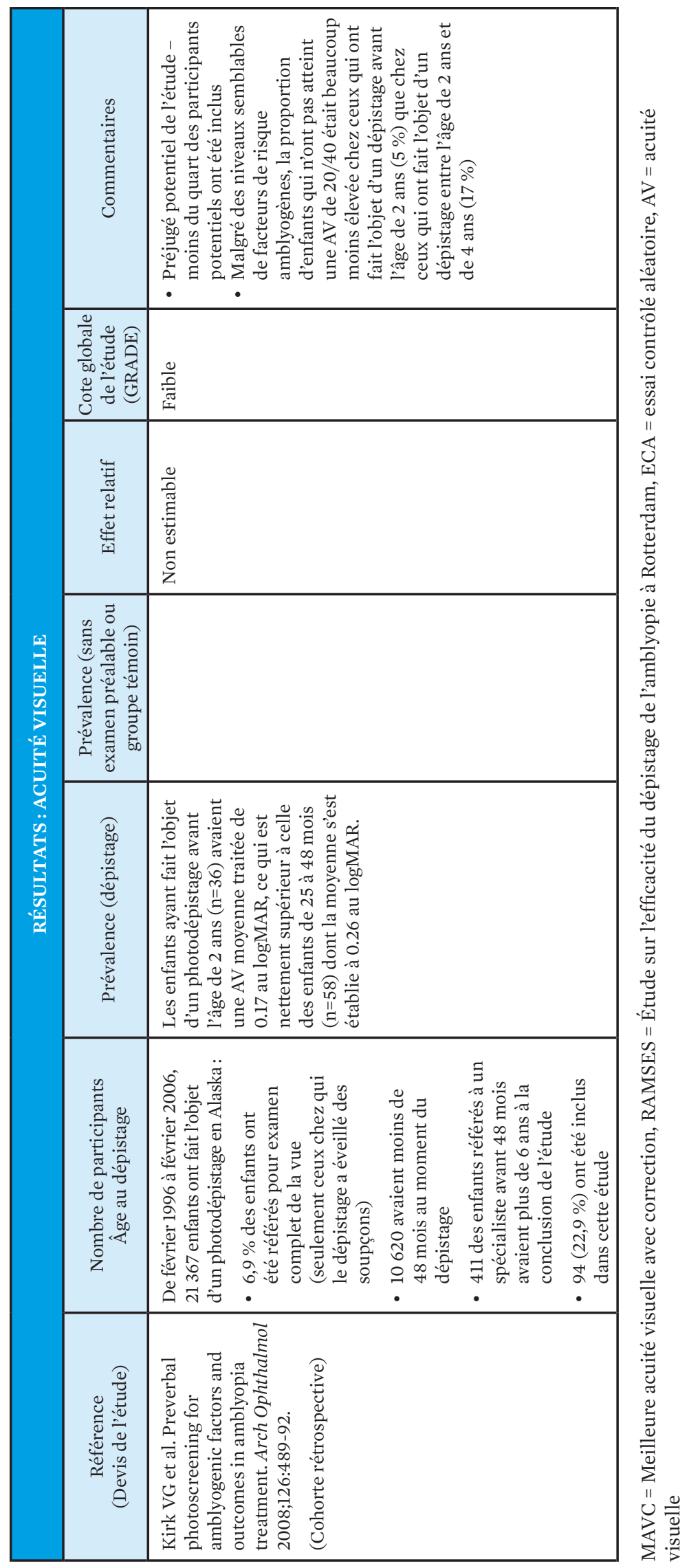

48

CANADIAN JOURNAL of OPTOMETRY | REVUE CANADIENNE D’OPTOMÉtRIE VOL. 81 NO. 4 


\section{RÉFÉRENCES}

1. Robinson BE, Mairs K, Glenny C, Stolee P. An evidence-based guideline for the frequency of optometric eye examinations. Primary Health Care 2012:121; doi 10.4172/2167-1079.1000121.

2. Comité d'experts sur les guides de pratique clinique. Canadian Ophthalmological Society evidence-based clinical practice guidelines for the periodic eye examination in adults in Canada. Can J Ophthalmol 2007;42:39-45.

3. Amit M; Société canadienne de pédiatrie, Comité de pédiatrie communautaire. Vision screening in infants, children and youth. Paediatr Child Health 2009; 14:246-51. Réaffirmé le $1^{\text {er }}$ février 2016 , https://www.cps.ca/fr/documents/position/children-vision-screening [consulté le 27 août 2019].

4. Dunfield L., Keating T. Preschool vision screening [rapport technique $\mathrm{n}^{\circ}$ 73]. Ottawa (Ont.) : Agence canadienne des médicaments et des technologies de la santé; 2007.

5. US Preventive Services Task Force; Grossman DC, Curry SJ, Owens DK, Barry MJ, Davidson KW, Doubeni CA et coll. Vision screening in children aged 6 months to 5 years: US Preventive Services Task Force recommendation statement. JAMA 2017;318:836-44.

6. American Academy of Ophthalmology Pediatric Ophthalmology/ strabismus Panel. Pediatric Eye Evaluations Preferred Practice Pattern $^{\circledR}$ : I. Vision screening in the primary care and community setting; II. Comprehensive ophthalmic examination. San Francisco (CA) : American Academy of Ophthalmology; 2012.

7. American Association for Pediatric Ophthalmology and Strabismus. Vision screening recommendations, https://aapos.org//client_data/ files/2014/1076_aapos_visscreen.pdf [consulté le 27 août 2019].

8. Rourke Baby Record. Evidence-based infant/child health maintenance guide, www.rourkebabyrecord.ca; 2017 [consulté le 27 août 2019].

9. ABCDaire. Recommandations concernant le dépistage des trouble de la vue chez les nourrissons et les enfants.https://enseignement. chusj.org/ENSEIGNEMENT/files/e7/e77f612e-86b5-4e5d-a5d4950459006a81.pdf [consulté 27 août 2019].

10. Jacobson PD. Transforming clinical practice guidelines into legislative mandates: proceed with abundant caution. JAMA 2008;299:208-10.

11. Davis D, Goldman J, Palda VA. Handbook on clinical practice guidelines. Ottawa (Ont.); Association médicale canadienne; 2007.

12. Association canadienne de protection médicale. Clinical practice guidelines: what is their role in legal proceedings? CMPA Perspective 2011;septembre:3-5.

13. AGREE Enterprise. Appraisal of guidelines research and evaluation II (AGREE II) instrument, http://www.agreetrust.org [consulté le 27 août 2019].

14. Brouwers M, Kho ME, Browman GP, Cluzeau F, Feder G, Fervers B, et coll. Consortium AGREE Next Steps. AGREE II: advancing guideline development, reporting and evaluation in healthcare. CMAJ 2010;182:E839-42.

15. Schünemann HJ, Wiercioch W, Etxeandia I, Falavigna M, Santesso N, Mustafa R, et coll. Guidelines 2.0: systematic development of a comprehensive checklist for a successful guideline enterprise. CMAJ 2014;186:E123-42.

16. Guyatt G, Oxman AD, Akl EA, Kunz R, Vist G, Brozek J et coll. GRADE guidelines: 1. Introduction - GRADE evidence profiles and summary of findings tables. J Clin Epidemiol 2011:64:383-94.

17. Groupe de travail du profil GRADE. http://www.gradeworkinggroup.org/; 2017 [consulté le 27 août 2019].

18. Guyatt GH, Oxman AD, Vist GE, Kunz R, Falck-Ytter Y, AlonsoCoello P et coll.; GRADE Working Group. GRADE: an emerging consensus on rating quality evidence and strength of recommendations. BMJ 2008; 336:924-6.

19. Fletcher RH, Fletcher SW, Fletcher GS. Clinical Epidemiology: The Essentials. 5e édition. Baltimore; Lippincott Williams \& Wilkins:2014:227.

20. Solebo AL, Cumberland PM, Rahi JS. Whole-population vision screening in children aged $4-5$ years to detect amblyopia. Lancet 2015;385:2308-19.

21. Tarczy-Hornoch K, Varma R, Cotter SA, McKean-Cowdin R, Lin JH, Borchert MS, et coll.; Joint Writing Committee for the Multi-Ethnic Pediatric Eye Disease Study and the Baltimore Pediatric Eye Disease Study Groups. Risk factors for decreased visual acuity in preschool children: the Multi-Ethnic Pediatric Eye Disease and Baltimore Pediatric Eye Disease Studies. Ophthalmology 2011;118:2262-73.
22. Pai AS, Wang JJ, Samarawickrama C, Burlutsky G, Rose KA, Varma R et coll. Prevalence and risk factors for visual impairment in preschool children in the Sydney Paediatric Eye Disease Study. Ophthalmology 2001;188:495-500.

23. Drover JR, Kean PG, Courage ML, Adams RJ. Prevalence of amblyopia and other vision disorders in young Newfoundland and Labrador children. Can J Ophthalmol 2008;43:89-94.

24. Irving EL, Harris JD, Machan CM, Robinson BE, Hrynchak PK, Leat SJ et coll. Value of routine eye examinations in asymptomatic patients. Optom Vis Sci 2016;93:660-6.

25. Huang J, Maguire MG, Ciner E, Kulp MT, Cyert LA, Quinn GE et coll.; Vision in Preschoolers Study Group. Risk factors for astigmatism in the Vision in Preschoolers Study. Optom Vis Sci 2014;91:514-21.

26. Pascual M, Huang J, Maguire MG, Kulp MT, Quinn GE, Ciner E et coll. Vision In preschoolers Study Group. Risk factors for amblyopia in the Vision In Preschoolers study. Ophthalmology 2014;121:622-9.

27. Varma R, Tarczy-Hornoch K, Jiang X. Visual impairment in preschool children in the United States: demographic and geographic variations from 2015 to 2060. JAMA Ophthalmol 2017;135(8):610-6.

28. Hendler K, Mehravaran S, Lu X, Brown SI, Mondino BJ, Coleman AL. Refractive errors and amblyopia in the UCLA Preschool Vision Program: first year results. Am J Ophthalmol 2016;172:80-6.

29. Attebo K, Mitchell P, Cumming R, Smith W, Jolly N, Sparkes R. Prevalence and causes of amblyopia in an adult population. Ophthalmology 1998;105:154-9.

30. Friedman DS, Repka MX, Katz J, Giordano L, Ibironke J, Hawse P et coll. Prevalence of amblyopia and strabismus in white and African American children aged 6 through 71 months. The Baltimore Pediatric Eye Disease Study. Ophthalmology 2009;116:2128-34.

31. Pai AS, Rose KA, Leone JF, Sharbini S, Burlutsky G, Varma R et coll. Amblyopia prevalence and risk factors in Australian preschool children. Ophthalmology 2012;119:138-44.

32. McKean-Cowdin R, Varma R, Cotter SA, Tarczy-Hornoch K, Borchert MS, Lin JH et coll. Multiethnic Pediatric Eye Disease Study and the Baltimore Pediatric Eye Disease Study Groups. Risk factors for astigmatism in preschool children: the Multi-Ethnic Pediatric Eye Disease and Baltimore Pediatric Eye Disease studies Ophthalmology 2011;118:1974-81.

33. Wallace DK, Repka MX, Lee KA, Melia M, Christiansen SP, Morse CL et coll. American Academy of Pediatric Ophthalmology/ strabismus Preferred Practice Pattern Pediatric Ophthalmology Panel. Amblyopia Preferred Practice Pattern ${ }^{\circledast}$. Ophthalmology 2018;125:105-42.

34. González EG, Wong AMF, Niechwiej-Szwedo E, Tarita-Nistor L, Steinbach MJ. Eye position stability in amblyopia and in normal binocular vision. Invest Ophthalmol Vis Sci 2012;53:5386-94.

35. Meier K, Giaschi D. Unilateral amblyopia affects two eyes: fellow eye deficits in amblyopia. Invest Ophthalmol Vis Sci 2017;58:1779-1800.

36. Hubel DH, Wiesel TN. Receptive fields, binocular interaction and functional architecture in the cat's visual cortex. $J$ Physiol 1962;106:106-54.

37. Assaf AA. The sensitive period: transfer of fixation after occlusion for strabismic amblyopia. Br J Ophthalmol 1982;66:64-70.

38. Wiesel TN, Hubel DH. Single-cell responses in striate cortex of kittens deprived of vision in one eye. $J$ Neurophysiol 1963;26:1003-17.

39. Tailor V, Bossi M, Bunce C, Greenwood JA, Dahlmann-Noor A. Binocular versus standard occlusion or blurring treatment for unilateral amblyopia in children aged three to eight years. Cochrane Database Syst Rev 2015;8:CD011347.

40. Sato M, Stryker MP. Distinctive features of adult ocular dominance plasticity. J Neurosci 2008;28:10278-86.

41. Evans BJ, Yu CS, Massa E, Mathews JE. Randomised controlled trial of intermittent photic stimulation for treating amblyopia in older children and adults. Ophthalmic Physiol Opt 2011;31:56-68.

42. Levi DM. Perceptual learning in adults with amblyopia: a reevaluation of critical periods in human vision. Dev Psychobiol 2005;46:222-32

43. Levi DM, Li RW. Improving the performance of the amblyopic visual system. Philos Trans R Soc Lond B Biol Sci 2009; 364:399-407.

44. Eibschitz-Tsimhoni M, Friedman T, Naor J, N, Friedman Z. Early screening for amblyogenic risk factors lowers the prevalence and severity of amblyopia. J AAPOS 2000;4:194-9. 
45. Pediatric Eye Disease Investigator Group. A randomized trial of atropine vs. patching for treatment of moderate amblyopia in children. Arch Ophthalmol 2002;120:268-78.

46. Barrett BT, Bradley A, Candy TR. The relationship between anisometropia and amblyopia. Prog Retin Eye Res 2013;36:120-58.

47. De Koning HJ, Groenewoud JH, Lantau VK, Tijam AM, Hoogeveen WC, de Faber JT et coll. Effectiveness of screening for amblyopia and other eye disorders in a prospective birth cohort study. J Med Screen 2013;20:66-72.

48. Williams C, Northstone K, Harrad RA, Sparrow JM, Harvey I; équipe de l'étude ALSPAC. Amblyopia treatment outcome after preschool screening $\mathrm{v}$ school entry screening: observational data for a prospective cohort study. Br J Ophthalmol 2003;87:988-93.

49. Williams C, Northstone K, Harrad RA, Sparrow JM, Harvey I; équipe de l'étude ALSPAC. Amblyopia treatment outcomes after screening before or at age 3 years: follow up from randomised trial. BMJ 2002; 324:1549.

50. Kirk VG, Clausen MM, Armitage MD, Arnold RW. Preverbal photoscreening for amblyogenic factors and outcomes in amblyopia treatment: early objective screening and visual acuities. Arch Ophthalmol 2008;126:489-92.

51. Groenewoud JH, Tjiam AM, Lantau VK, Hoogeveen WC, de Faber JT, Juttmann RE et coll. Rotterdam amblyopia Screening Effectiveness Study : detection and causes of amblyopia in a large birth cohort. Invest Ophthalmol Vis Sci 2010;51:3476-84.

52. VIP Study Group. Does assessing eye alignment along with refractive error or visual acuity increase sensitivity for detection of strabismus in preschool vision screening? Invest Ophthalmol Vis Sci 2007;48:3115-25.

53. Atkinson J, Braddick O, Nardini M, Anker S. Infant hyperopia: detection, distribution, changes and correlates - outcomes from the Cambridge Infant Screening Programs. Optom Vis Sci 2007;84:84-96.

54. Membreno JH, Brown MM, Brown GC, Sharma S, Beauchamp GR. A cost-utility analysis of therapy for amblyopia. Ophthalmology 2002;109:2265-71.

55. Konig HH, Barry JC. Cost-effectiveness of treatment for amblyopia: an analysis based on a probabilistic Markov Model. Br J Ophthalmol Mai 2004;88:606-12.

56. Holmes JM, Beck RW, Kraker RT, Astle WF, Birch EE, Cole SR et coll.; Pediatric Eye Disease Investigator Group. Risk of amblyopia recurrence after cessation of treatment. J AAPOS 2004;8:420-8.

57. Holmes JM, Melia M, Bradfield S, Cruz OA, Forbes B; Pediatric Eye Disease Investigator Group. Factors associated with recurrence of amblyopia on cessation of patching. Ophthalmology 2007;114:1427-32.

58. Von Noorden GK, Dowling JE. Experimental amblyopia in monkeys. II. Behavioral studies in strabismic amblyopia. Arch Ophthalmol 1970;84:215-20.
59. Baker FH, Grigg P, von Noorden GK. Effects of visual deprivation and strabismus on the response of neurons in the visual cortex of the monkey, including studies of striate and prestriate cortex in the normal animal. Brain Res 1974;66:185-208.

60. Hess RF, Thompson B. Amblyopia and the binocular approach to its therapy. Vision Res 2015; 114:4-16.

61. Birch EE, Li SL, Jost RM, Morale SE, De La Cruz A, Stager D Jr et coll. Binocular iPad treatment for amblyopia in preschool children. $J$ AAPOS 2015;19:6-11.

62. American Academy of Pediatrics, Comité sur la pratique et la médecine ambulatoire, Section de l'ophtalmologie; American Association of Certified Orthoptists; American Association for Pediatric Ophthalmology and strabismus; American Academy of Ophthalmology. Eye examination in infants, children and young adults by pediatricians. Pediatrics 2003;111:902-7.

63. Le TD, Raashid RA, Colpa L, Noble J, Ali A, Wong A. Paediatric vision screening in the primary care setting in Ontario. Paediatr Child Health 2018;23:e33-9.

64. Lennerstrand G, Jakobsson P, Kvarnström G. Screening for ocular dysfunction in children: approaching a common program. Acta Ophthalmol Scand Suppl 1995;214:26-38.

65. Schmidt P, Maguire M, Dobson V, Quinn G, Ciner E, Cyert L et coll.; Vision in Preschoolers Study Group. Comparison of preschool vision screening tests as administered by licensed eye care professionals in the Vision in Preschoolers Study. Ophthalmology 2004;111:637-50

66. American Optometric Association. Comprehensive pediatric eye and vision examination: evidence-based clinical practice guideline. 2017:1-67. https://www.aoa.org/optometrists/tools-and-resources/ evidence-based-optometry/evidence-based-clinical-practice-guidlines/evidence-based-clinical-practice-guideline-comprehensivepediatric-eye-and-vision-examination [consulté le 27 août 2019].

67. Goldbloom R. Pediatric clinical skills. $4^{\mathrm{e}}$ éd. Philadelphie (PA) : Saunders; 2010.

68. Sloot F, Sami A, Karaman H, Benjamins J, Loudon SE, Raat H et coll. Effect of omission of population-based eye screening at age 6-9 months in the Netherlands. Acta Ophthalmol 2015;93:318-21.

69. Donahue SP. Relationship between anisometropia, patient age, and the development of amblyopia. Am J Ophthalmol 2006;142:132-40.

70. Zaba JH, Reynolds W, Mozlin R, Costich J, Slavova S, Steele GT. Comparing the effectiveness of vision screenings as part of the school entrance physical examinations in children ages 3 to 6: an exploratory study. Optometry 2007;78:514-22. 